TRANSACTIONS OF THE

AMERICAN MATHEMATICAL SOCIETY

Volume 365, Number 2, February 2013, Pages 973-999

S 0002-9947(2012)05657-1

Article electronically published on July 25, 2012

\title{
SMALL OPTIMAL MARGULIS NUMBERS FORCE UPPER VOLUME BOUNDS
}

\author{
PETER B. SHALEN
}

Dedicated to José Montesinos on the occasion of his 65th birthday

Abstract. If $\lambda$ is a positive real number strictly less than $\log 3$, there is a positive number $V_{\lambda}$ such that every orientable hyperbolic 3-manifold of volume greater than $V_{\lambda}$ admits $\lambda$ as a Margulis number. If $\lambda<(\log 3) / 2$, such a $V_{\lambda}$ can be specified explicitly and is bounded above by

$$
\lambda\left(6+\frac{880}{\log 3-2 \lambda} \log \frac{1}{\log 3-2 \lambda}\right),
$$

where log denotes the natural logarithm. These results imply that for $\lambda<\log 3$, an orientable hyperbolic 3-manifold that does not have $\lambda$ as a Margulis number has a rank-2 subgroup of bounded index in its fundamental group and in particular has a fundamental group of bounded rank. Again, the bounds in these corollaries can be made explicit if $\lambda<(\log 3) / 2$.

\section{INTRODUCTION}

Let $M$ be a (complete) orientable hyperbolic 3-manifold. Up to isometry, we may identify $M$ with $\mathbb{H}^{3} / \Gamma$, where $\Gamma$ is a discrete, torsion-free subgroup of $\operatorname{Isom}_{+}\left(\mathbb{H}^{3}\right)$, uniquely determined up to conjugacy by the hyperbolic structure of $M$ and isomorphic to $\pi_{1}(M)$. In this setting we have the following definition:

Definition 1.1. A Margulis number for $M$ (or for $\Gamma$ ) is a positive real number $\mu$ such that the following condition holds:

1.0.1. If $P$ is a point of $\mathbb{H}^{3}$ and $x, y$ are elements of $\Gamma$ such that

$$
\max (d(P, x \cdot P), d(P, y \cdot P))<\mu,
$$

then $x$ and $y$ commute.

Here, and throughout this paper, $d$ denotes hyperbolic distance on $\mathbb{H}^{3}$.

We refer the reader to the introduction of 32 for a general background discussion of Margulis numbers, including external references generalizations to the case of higher-dimensional or non-orientable hyperbolic manifolds. In 32 we pointed out that if $\Gamma \cong \pi_{1}(M)$ is non-abelian, then there is an optimal Margulis number for $M$, denoted $\mu(M)$, characterized by the property that a given positive number $\mu$ is a Margulis number for $M$ if and only if $\mu \leq \mu(M)$. We also discussed the Margulis Lemma, which implies that there is a constant which is a Margulis number for

Received by the editors October 13, 2010 and, in revised form, June 16, 2011.

2010 Mathematics Subject Classification. Primary 57M50.

This work was partially supported by NSF grant DMS-0906155.

(C) 2012 American Mathematical Society 
every orientable hyperbolic 3-manifold. In this paper we will denote the largest such constant by $\mu_{+}(3)$.

In 32 we discussed the problem of finding lower bounds for $\mu(M)$, as $M$ varies over a prescribed class of orientable hyperbolic manifolds, and its significance for the problem of classifying finite-volume orientable hyperbolic 3-manifolds. Results on this problem include Meyerhoff's lower bound of $0.104 \ldots$ for $\mu_{+}(3)$, given in 24] (which should be compared with Marc Culler's upper bound of $0.616 \ldots$...) the main result of 9 , which implies that $\mu(M) \geq 0.286$ for any orientable hyperbolic Haken manifold $M$; the main result of 32], which asserts that, up to isometry, there are at most finitely many orientable 3 -manifolds with $\mu(M)<0.29$; and Corollaries 3.13 and 3.14 of this paper, which assert that if every subgroup of rank at most 2 in $\pi_{1}(M)$ has infinite index - and in particular if $H_{1}(M ; \mathbb{Q})$ has rank at least 3 or if $M$ is closed and $H_{1}\left(M ; \mathbb{Z}_{p}\right)$ has rank at least 4 for some prime $p$ then $\mu(M) \geq \log 3=1.09 \ldots$ (These corollaries are well-known consequences of known results and are included in the present paper for completeness. The essential ingredients are, first, the "log 3 theorem", which is deduced from the results of [10, [4, 1], 6], 28, and [29], and, second, some topological facts proved in [17] and [34.)

It is a standard observation that a lower bound for $\mu(M)$ forces a lower bound for the volume of $M$. Indeed, if $\operatorname{vol} M<\infty$, it is easy to deduce that the $\mu$-thick part of $M[5$, Chapter $\mathrm{D}]$ is non-empty and hence that $M$ contains an isometric copy of a ball $B$ of radius $\mu / 2$ in $\mathbb{H}^{3}$. In particular, the volume of $B$ is a lower bound for vol $M$. Various refinements of this estimate are also well known.

The theme of the present paper, exemplified by Theorems A and B below and their corollaries, is that for an orientable hyperbolic 3-manifold $M$, certain upper bounds on $\mu(M)$ force upper bounds on the volume of $M$. From this one can deduce that certain upper bounds on $\mu(M)$ also force upper bounds on certain group-theoretical invariants of $\pi_{1}(M)$ such as its rank.

Of course, these results can be reinterpreted as saying that suitable lower bounds on the volume of $M$ or on such group-theoretical invariants as the rank of $\pi_{1}(M)$ imply certain lower bounds on $\mu(M)$. From this point of view, the paper can be seen as a contribution to a body of results, discussed above, that give lower bounds for $\mu(M)$ under various restrictions on $M$.

The following result, which gives a first illustration of how upper bounds on $\mu(M)$ force upper bounds on the volume of $M$, will be proved in the body of the paper as Theorem 4.2 .

Theorem A. Let $\lambda$ be a positive real number strictly less than $\log 3$. Then there is a constant $V_{\lambda}$ such that for every orientable hyperbolic 3-manifold $M$ with $\mu(M)<\lambda$ we have vol $M \leq V_{\lambda}$ (and in particular vol $M<\infty$ ).

The following two corollaries to Theorem A will be proved in the body of the paper as Corollaries 4.1 and 4.2 .

Corollary. Let $\lambda$ be a positive real number strictly less than $\log 3$. Then there is a natural number $d_{\lambda}$ such that for every orientable hyperbolic 3-manifold $M$ with $\mu(M)<\lambda$, the group $\pi_{1}(M)$ has a rank-2 subgroup of index at most $d_{\lambda}$.

Corollary. Let $\lambda$ be a positive real number strictly less than $\log 3$. Then there is a natural number $k_{\lambda}$ such that for every orientable hyperbolic 3-manifold $M$ with $\mu(M)<\lambda$, the group $\pi_{1}(M)$ has rank at most $k_{\lambda}$. 
Note that in Theorem A and the two Corollaries stated above, no explicit estimate is given for the constants $V_{\lambda}, d_{\lambda}$ and $k_{\lambda}$. As we shall now explain, explicit estimates can be obtained if we replace the assumption $\lambda<\log 3$ by the stronger assumption that $\lambda<(\log 3) / 2$.

The following result will be proved in the body of the paper as Corollary 7.3 . (It is a corollary to a more technical result, Theorem 7.1.)

Theorem B. Let $\lambda$ be a positive real number strictly less than $(\log 3) / 2$. Then for every orientable hyperbolic 3-manifold $M$ with $\mu(M)<\lambda$ we have

$$
\operatorname{vol} M<\lambda\left(6+\frac{880}{\log 3-2 \lambda} \log \frac{1}{\log 3-2 \lambda}\right) .
$$

To avoid confusion it may be worth pointing out that the right hand side of the inequality in the conclusion of Theorem B is negative if, say, $\lambda<0.1$. Thus in this case the theorem asserts that $\mu(M)$ cannot be less than $\lambda$. However, this is not new information, as Meyerhoff [24] has shown that $\mu(3)>0.1$, and indeed his result is used in the proof of Theorem B.

The following two corollaries to Theorem B will be proved in the body of the paper as Corollaries 7.4 and 7.5 .

Corollary. Let $\lambda$ be a positive real number strictly less than $(\log 3) / 2$. Then for every orientable hyperbolic 3-manifold $M$ with $\mu(M)<\lambda$, the group $\pi_{1}(M)$ has a rank-2 subgroup of index at most

$$
\frac{\lambda}{V_{0}}\left(6+\frac{880}{\log 3-2 \lambda} \log \frac{1}{\log 3-2 \lambda}\right) .
$$

Corollary. Let $\lambda$ be a positive real number strictly less than $(\log 3) / 2$. Then for every hyperbolic 3-manifold $M$ with $\mu(M)<\lambda$, we have

$$
\operatorname{rank} \pi_{1}(M) \leq 2+\log _{2}\left(\frac{\lambda}{V_{0}}\left(6+\frac{880}{\log 3-2 \lambda} \log \frac{1}{\log 3-2 \lambda}\right)\right) .
$$

In the author's forthcoming paper [33, a refined version of Theorem A will be used to prove the following result:

Theorem. Let $K$ be a number field. Then up to isometry there are at most finitely many closed, orientable hyperbolic 3-manifolds that have trace field $K$ and do not admit 0.34 as a Margulis number.

In another work of the author's, not yet written, Theorem 7.1 is combined with arguments invoking many other results - the $\log 3$ theorem, the algebra of congruence subgroups, and Beukers and Schlickewei's explicit form of Siegel and Mahler's finiteness theorem for solutions to the unit equation in number fields - to prove the following result:

Theorem. Let $K$ be any number field, and let $D$ denote its degree. The number of (isometry classes of) closed, non-arithmetic hyperbolic 3-manifolds which are $\mathbb{Z}_{6}$-homology 3-spheres, have trace field $K$, and have optimal Margulis number less than 0.183 is at most $141 \times 2^{24(D+1)}$.

(That the number of such isometry classes is finite follows from the main result of [32. It is the explicit bound which is the content of the theorem above.)

The proof of Theorem A occupies Section 4. The method of proof is to reduce the result to the $\log 3$ theorem (which was mentioned above) using relatively formal 
arguments based on standard results about algebraic and geometric convergence. The basic strategy is similar to the one used in 32 .

The proof of Theorem B is rather easily reduced to the case in which $\pi_{1}(M)$ is a two-generator group. In this case, the proof involves two steps. The first, which is carried out in Section 5, consists of showing that an upper bound $\lambda<(\log 3) / 2$ on $\mu(M)$ forces an explicit upper bound on the minimal length of a non-trivial relation in the generators of $\pi_{1}(M)$. This step, which is embodied in Proposition 5.3, is a refinement of the elementary packing arguments that are used, for example, in 34 to give an elementary proof that $(\log 3) / 2$ is a Margulis number for any hyperbolic 3-manifold $M$ such that every two-generator subgroup of $\pi_{1}(M)$ is free.

The second step involved in proving Theorem $\mathrm{B}$ in the case in which $\pi_{1}(M)$ is a two-generator group is to show that an upper bound on the minimal length of a non-trivial relation in the generators of $\pi_{1}(M)$ forces an explicit upper bound on the volume of $M$. This bound is given by Proposition 6.2 the proof of which is the goal of Section 6 The proof of Proposition 6.2 is a refinement, in the two-generator case, of the argument used by Cooper in 8 to show that the volume of a closed hyperbolic 3-manifold is bounded by $\pi$ times the length of a presentation of its fundamental group. Adapting Cooper's method to give a volume bound in terms of the length of a single relation rather than the length of an entire presentation involves some ingredients which are required but did not appear in [8]. One of these is an isoperimetric inequality which was proved by Agol and Liu as Lemma 4.4 of their paper [3], where they applied it to prove a result that is somewhat analogous to, but distinct from, Proposition 6.2.

The newest ingredient needed for the proof of Proposition 6.2 is a deep and technical topological result, Lemma 6.2. One of the results needed to prove Lemma 6.2. Proposition 6.1, is a new application of the characteristic submanifold theory that seems to be of particular independent interest.

In Section 17 we assemble the results of Sections 5 and 6 to prove Theorem 7.1 and its various corollaries (including Corollary 7.3 which is Theorem B above).

Sections 2 and 3 are preliminary sections, devoted to assembling more or less well-known results used in later sections for which convenient references are not easy to find.

The author is grateful to Michael Siler, Dick Canary and Marc Culler for a series of valuable discussions on the material in this paper. Siler pointed out an error in an earlier version of the paper; he called attention to Lemma 4.4 of Agol and Liu's paper [3] and most importantly he made a suggestion that led to the realization that $\pi$ could be replaced by $\min (\pi, \lambda)$ in Proposition 6.2. which gives an important improvement in Theorem 7.1. Canary has painstakingly continued to educate the author about algebraic and geometric convergence; he corrected naïve ideas about the algebraic convergence arguments involved in the proof of Theorem 4.2 and helped the author to the correct argument and suitable references. Culler patiently listened to all the details of the material in the paper before they were written and clarified the proof of Proposition 2.2 .

\section{General Preliminaries}

Throughout this paper, $\log x$ will denote the natural logarithm of a positive number $x$ and $\#(X)$ will denote the cardinality of a finite set $X$. 
In statements and arguments involving fundamental groups, we will suppress base points whenever it is possible to do so without ambiguity. If $X$ is a pathconnected space, we will often implicitly assume that $X$ is equipped with an unnamed (and arbitrary) base point $\star_{X}$ and write $\pi_{1}(X)$ for $\pi_{1}\left(X, \star_{X}\right)$. If $f: X \rightarrow Y$ is a map between path-connected spaces, then $f_{\sharp}: \pi_{1}(X) \rightarrow \pi_{1}(Y)$ will mean the homomorphism from $\pi_{1}\left(X, \star_{X}\right)$ to $\pi_{1}(Y)=\pi_{1}\left(Y, \star_{Y}\right)$ which is obtained by composing the standard induced homomorphism $f_{\sharp}: \pi_{1}\left(X, \star_{X}\right) \rightarrow \pi_{1}\left(Y, f\left(\star_{X}\right)\right)$ with the isomorphism from $\pi_{1}\left(Y, f\left(\star_{X}\right)\right)$ to $\pi_{1}\left(Y, \star_{Y}\right)$ defined by an unspecified path from $f\left(\star_{X}\right)$ to $\star_{Y}$. Thus $f_{\sharp}$ is well defined up to post-composition with inner isomorphisms of $\pi_{1}(Y)$. Many assertions about $f_{\sharp}$, such as the assertion that is injective or surjective, are invariant under post-composition with inner isomorphisms and will be made without reference to a connecting path. Likewise, the image of an element or subgroup of $\pi_{1}(X)$ under $f_{\sharp}$ is well defined up to conjugacy.

A path-connected subset $A$ of a path-connected space $X$ will be termed $\pi_{1}$ injective if the inclusion homomorphism $\pi_{1}(A) \rightarrow \pi_{1}(X)$ is injective.

The following two easy results from group theory will be needed later in the paper.

Lemma 2.1. Let $F$ be a free group on a generating set $S$, let $Z$ be a cyclic subgroup of $F$, and let $k$ be a positive integer. Then there are at most $2 k+1$ elements of $Z$ that can be expressed as words of length at most $k$ in the generating set $S$.

Proof. Let $t$ be a generator of $Z$. If $t=1$ the assertion is trivial. If $t \neq 1$, there exist a reduced word $V$ in the generating set $S$ and a cyclically reduced word $W$ in $S$ such that the word $V * W * \bar{V}$ is reduced and represents $t$; here $*$ denotes concatenation of words and $\bar{V}$ denotes the inverse of the word $V$. For any non-zero integer $n$ the word $V *\left(\star^{n} W\right) * \bar{V}$ is reduced and represents $t^{n}$; here $\star^{n} W$ denotes the $n$-fold concatenation of $W$. In particular, the unique reduced word representing $t^{n}$ has length at least $n$. If $t^{n}$ can be expressed as a word of length at most $k$, then the reduced word represented $t^{n}$ has length at most $k$, and hence $n \leq k$. The conclusion follows.

Proposition 2.1. Suppose that $\widetilde{\Gamma}$ is a finite-index subgroup of a finitely generated group $\Gamma$. Then

$$
\operatorname{rank} \Gamma \leq \operatorname{rank} \widetilde{\Gamma}+\log _{2}[\Gamma: \widetilde{\Gamma}]
$$

Proof. Set $r=\operatorname{rank} \widetilde{\Gamma}$ and fix a generating set $\widetilde{S}$ for $\widetilde{\Gamma}$ with $|\widetilde{S}|=r$. Let $S \supset \widetilde{S}$ be a finite generating set for $\Gamma$, and let $S$ be chosen to have minimal cardinality among all generating sets for $\Gamma$ that contain $S$. Let us denote the distinct elements of $S-\widetilde{S}$ by $x_{1}, \ldots, x_{k}$. For $0 \leq j \leq k$, let $\Gamma_{j}$ denote the subgroup of $\Gamma$ generated by $\widetilde{S} \cup\left\{x_{1}, \ldots, x_{j}\right\}$ (so that in particular $\Gamma_{0}=\widetilde{\Gamma}$ and $\Gamma_{k}=\Gamma$ ). It follows from the minimality of $S$ that $\Gamma_{j-1}$ is a proper subgroup of $\Gamma_{j}$ for $j=1, \ldots, k$, and therefore $\left[\Gamma_{j}: \Gamma_{j-1}\right] \geq 2$. Hence

$$
[\Gamma: \widetilde{\Gamma}]=\prod_{j=1}^{k}\left[\Gamma_{j}: \Gamma_{j-1}\right] \geq 2^{k}
$$

Using this, we find

$$
\operatorname{rank} \Gamma \leq|S|=r+k \leq r+\log _{2}[\Gamma: \widetilde{\Gamma}]
$$


The following elementary fact from hyperbolic geometry will also be needed.

Proposition 2.2. Let $T$ be a triangle in a hyperbolic space, and let $L$ denote the length of the shortest side of $T$. Then

$$
\text { area } T<\min (\pi, L) \text {. }
$$

Proof. We may assume that $T \subset \mathbb{H}^{2}$. Let $l$ denote a side of $T$ having length $L$, and let $\overline{\mathbb{H}}^{2}$ denote the union of $\mathbb{H}^{2}$ with the circle at infinity. There is a triangle $T^{\prime}$ in $\overline{\mathbb{H}}^{2}$ which contains $T$, has $l$ as a side, and has an ideal vertex opposite $l$. It is enough to prove that area $T^{\prime}<\min (\pi, L)$. Let us identify $\mathbb{H}^{2}$ with the upper half-plane model in such a way that $l$ is an arc in the upper unit semicircle and the other two sides of $T^{\prime}$ are vertical rays. Let $\left(x_{1}, y_{1}\right)$ and $\left(x_{2}, y_{2}\right)$ be the endpoints of $l$ in Cartesian coordinates, where $-1<x_{1}<x_{2}<1$. We have

$$
\operatorname{area} T^{\prime}=\int_{x_{1}}^{x_{2}} \int_{\sqrt{1-x^{2}}}^{\infty} \frac{1}{y^{2}} d y d x=\arcsin x_{2}-\arcsin x_{1},
$$

which is the Euclidean length of the arc $l$. This shows that area $T^{\prime}<\pi$. On the other hand, the hyperbolic length $L$ of $l$ is the integral over the arc $l$ of the hyperbolic length element, which is given by $d s / y$ where $d s$ is the Euclidean length element. Since $y<1$ at all but at most one point of the $\operatorname{arc} l$, the hyperbolic length of $l$ strictly exceeds its Euclidean length, so that area $T^{\prime}<L$.

\section{THREE-MANIFOLD PRELIMINARIES}

3.1. When no category is specified, it will be understood that "manifolds" and "submanifolds" are smooth. At various points in the paper we will need to mention PL or real-analtyic manifolds, and in these cases we will be explicit about the category in question.

As is customary in 3-manifold topology, we will frequently quote results about 3 -manifolds that are proved in the PL category and apply them in the smooth category, most often without explicitly mentioning the transition. In most cases this will be justified by the following facts:

(1) If $M$ is a smooth $n$-manifold and $V$ is a (possibly empty) smooth, properly embedded submanifold, then $M$ has a smooth triangulation with respect to which $V$ is a polyhedral subset.

(2) Any two smooth triangulations of a given smooth manifold determine the same PL structure up to PL homeomorphism.

(3) If $n \leq 3$ and if $M$ and $M^{\prime}$ are smooth $n$-manifolds which are PL homeomorphic with respect to the PL structures defined by smooth triangulations, then $M$ and $M^{\prime}$ are diffeomorphic.

Of these facts, (2), and the case of (1) where $V=\emptyset$, are proved in [37, and (3) is included in [27, Theorem 6.3]. We have not located a direct reference for the case of (11) in which $V \neq \emptyset$, but it is a very special case of the main result of [12.

In Section 6 we will use a somewhat different result about the interaction between the PL and smooth (or rather real-analytic) categories:

Proposition 3.2. Suppose that $M$ is a real-analytic manifold and that $X_{1}, \ldots, X_{n}$ are semi-analytic sets. Then $M$ admits a smooth triangulation with respect to which $X_{1}, \ldots, X_{n}$ are polyhedral subsets. 
Proof. It is shown in 13 that $M$ is real-analytically isomorphic to a real-analytic submanifold $M^{\prime}$ of $\mathbf{R}^{N}$ for some $N$. If we identify $M$ with $M^{\prime}$, the sets $M, X_{1}, \ldots$, $X_{n}$ become semi-analtyic subsets of $\mathbf{R}^{N}$. The main theorem of [14 then asserts that $\mathbf{R}^{N}$ admits a smooth triangulation with respect to which $M, X_{1}, \ldots, X_{n}$ are polyhedral subsets. The conclusion follows.

Corollary 3.3. Let $M$ be a hyperbolic n-manifold, let $p: \mathbb{H}^{n} \rightarrow M$ be a locally isometric covering map, and let $\delta_{1}, \ldots, \delta_{k}$ be closed hyperbolic simplices in $\mathbb{H}^{n}$. Then $M$ admits a smooth triangulation with respect to which $X_{1}, \ldots, X_{n}$ are polyhedral subsets.

Proof. Each $\delta_{i}$ may be subdivided into finitely many closed hyperbolic simplices which are embedded in $M$ under $p$. Hence we may assume without loss of generality that the $\delta_{i}$ are themselves embedded in $M$ under $p$. In this case the sets $p\left(\delta_{1}\right), \ldots, p\left(\delta_{k}\right)$ are clearly semi-analytic in the real analytic structure defined by the hyperbolic structure of $M$, and so the result follows from Proposition 3.2

We will say that an orientable 3 -manifold $M$ is irreducible if $M$ is connected and every (smooth) 2-sphere in $M$ bounds a (smooth) ball in $M$.

Lemma 3.1. Let $X_{0}$ be a compact, connected, 3-dimensional submanifold of an irreducible, orientable 3-manifold $M$. Then there is a compact, irreducible, 3dimensional submanifold $X_{1}$ of $M$ such that $X_{1} \supset X_{0}$ and such that the inclusion homomorphism $\pi_{1}\left(X_{0}\right) \rightarrow \pi_{1}\left(X_{1}\right)$ is surjective.

Proof. Let $\mathcal{X}$ denote the set of all compact 3-dimensional submanifolds $X$ of $M$ such that $X \supset X_{0}$ and the inclusion homomorphism $\pi_{1}\left(X_{0}\right) \rightarrow \pi_{1}(X)$ is surjective. We have $X_{0} \in \mathcal{X}$. Let $X_{1} \in \mathcal{X}$ be chosen to have the smallest number of boundary components among all submanifolds in $\mathcal{X}$. It suffices to show that $X_{1}$ is irreducible. If $S \subset$ int $X_{1}$ is a 2-sphere, then $S$ bounds a ball $B \subset Q$. If $B \not \subset X_{1}$, then $X_{1} \cup B$ is a compact submanifold of $Q$ containing $X_{1}$ and having fewer boundary components than $X_{1}$. It is clear that the inclusion homomorphism $\pi_{1}\left(X_{1}\right) \rightarrow \pi_{1}\left(X_{1} \cup B\right)$ is surjective and hence that $X_{1} \cup B \in \mathcal{X}$, a contradiction to minimality.

Definitions 3.4. Let $M$ be an irreducible, orientable 3 -manifold. Following the convention of [15], we will define an incompressible surface in $M$ to be a compact, properly embedded 2-manifold in $M$ which is $\pi_{1}$-injective, is not a sphere, and is not a disk which forms the frontier of a topological ball.

A closed (smooth) 2-manifold $S$ in int $M$ is said to be boundary-parallel if $S$ is the frontier of a (smooth) submanifold $H$ of $M$ such that the pair $(H, S)$ is diffeomorphic to $(S \times[0,1], S \times\{1\})$. (The definition in the case of a properly embedded surface with non-empty boundary would be slightly trickier in the smooth category, but will not be needed in this paper.)

We will define a Haken manifold to be a compact, irreducible, orientable 3manifold which contains an incompressible surface. Note that according to this definition a 3-ball is not a Haken manifold.

By an essential disk in the irreducible, orientable 3-manifold $M$ we will mean a properly embedded disk whose boundary does not bound a disk in $\partial M$. We will say that $M$ is boundary-irreducible if $M$ contains no essential disk. It follows from the Loop Theorem [15, p. 39, 4.2] that $M$ is boundary-irreducible if and only if every component $T$ of $\partial M$ is $\pi_{1}$-injective in $M$. 
Lemma 3.2. Let $N$ be an irreducible, orientable 3-manifold, let $T \subset \operatorname{int} M$ be a closed incompressible surface, and suppose that the inclusion map $T \rightarrow N$ is homotopic to a map of $T$ into $\partial N$. Then $T$ is boundary-parallel in $N$.

Proof. In the case where $N$ is compact this is included in [35, Lemma 5.3]. To prove it in the general case, note that since the inclusion map $i: T \rightarrow N$ is homotopic to some map $f$ of $T$ into $\partial N$, there is a compact subset $X_{0}$ of $N$, containing $T$ and $f(T)$, such that $i$ is homotopic to $f$ in $X_{0}$. After passing to a regular neighborhood we may assume that $X_{0}$ is a submanifold of $N$. According to Lemma 3.1, there is a compact irreducible submanifold $X_{1}$ of $M$ such that $X_{1} \supset X_{0}$. Applying the compact case of the lemma, with $X_{1}$ in place of $N$, we deduce that $T$ is boundary parallel in $X_{1}$, and hence in $N$.

Definition 3.3. Let $M$ be an orientable hyperbolic 3-manifold, and let $p: \mathbb{H}^{3} \rightarrow M$ be a locally isometric covering map. A $\mathbb{Z} \times \mathbb{Z}$-cusp neighborhood in $M$ is a subset $C$ of $M$ such that $p^{-1}(C) \subset \mathbb{H}^{3}$ is a horoball, and the image of the inclusion homomorphism $\pi_{1}(C) \rightarrow \pi_{1}(M)$ is a free abelian group of rank 2 .

Proposition 3.5. Let $M$ be an orientable hyperbolic 3-manifold. Then

(1) $M$ is aspherical and irreducible.

(2) Every incompressible torus $T \subset M$ is the boundary of a submanifold $C$ of $M$ which is closed as a subset of $M$, is diffeomorphic to $T^{2} \times[0, \infty)$, and has finite volume.

(3) For every torus $T \subset M$ which is not incompressible, either (a) $T$ is contained in a 3-ball in $M$ or (b) $T$ is the boundary of a solid torus in $M$.

Proof. Write $M=\mathbb{H}^{3} / \Gamma$ where $\Gamma \subset$ Isom $_{+}\left(\mathbb{H}^{3}\right)$ is discrete and torsion-free. Let $p: \mathbb{H}^{3} \rightarrow M$ denote the quotient map. Since the universal covering $\mathbb{H}^{3}$ of $M$ is contractible, $M$ is aspherical. To prove that $M$ is irreducible, suppose that $S \subset M$ is a 2 -sphere. Then $S$ lifts to a 2-sphere $\widetilde{S} \subset \mathbb{H}^{3}$. Since $\mathbb{H}^{3}$ is diffeomorphic to $\mathbf{R}^{3}$, it is irreducible by [26, Theorem 1], and so $\widetilde{S}$ bounds a ball $\widetilde{B} \subset \mathbb{H}^{3}$. For any $\gamma \in \Gamma-\{1\}$, we have $\widetilde{S} \cap \gamma \cdot \widetilde{S}=\emptyset$. By the Brouwer fixed point theorem we cannot have $\widetilde{B} \subset \gamma \cdot \widetilde{B}$ or $\widetilde{B} \supset \gamma \cdot \widetilde{B}$, and since $\mathbb{H}^{3}$ is non-compact we cannot have $\widetilde{B} \cup \gamma \cdot \widetilde{B}=\mathbb{H}^{3}$. Hence $\widetilde{B} \cap \gamma \cdot \widetilde{B}=\emptyset$ for every $\gamma \in \Gamma-\{1\}$. It follows that $B:=p(\widetilde{B})$ is a 3 -ball with boundary $S$, and the proof of (11) is complete.

To prove (2), consider a torus $T \subset M$ which is incompressible. The image of the inclusion homomorphism $\pi_{1}(T) \rightarrow \pi_{1}(M)$ is a rank-2 free abelian subgroup of $\pi_{1}(M)$ which is defined up to conjugacy and corresponds to a rank-2 free abelian subgroup $X$ of $\Gamma$ which is also defined up to conjugacy. Since $\Gamma$ is discrete, $X$ must be parabolic. It is then a standard consequence of Shimizu's lemma (see for example [31, Theorem 2.21]) that there is a horoball $H \subset \mathbb{H}^{3}$, precisely invariant under $\Gamma$, whose stabilizer $\Gamma_{H}$ contains $X$. In particular $\Gamma_{H}$ is non-cyclic, and since it is parabolic and torsion-free it must be a rank-2 free abelian group. Hence $C_{0}:=H / \Gamma$ is a $\mathbb{Z} \times \mathbb{Z}$-cusp neighborhood. After possibly replacing $C_{0}$ by a smaller $\mathbb{Z} \times \mathbb{Z}$-cusp neighborhood, we may assume that $C_{0} \cap T=\emptyset$. Since $X \leq \Gamma_{H}$ and since $M$ is aspherical by (10), the inclusion map of $T$ into $M$ is homotopic in $M$ to a map whose image is contained in $C_{0}$. This inclusion map is therefore homotopic in $\overline{M-C_{0}}$ to a map whose image is contained in $\partial C_{0}$. It now follows from Lemma 3.2 . applied with $N=\overline{M-C_{0}}$, that $T$ is boundary-parallel in $\overline{M-C_{0}}$; that is, there is a submanifold $P$ of $M$, diffeomorphic to $T^{2} \times[0,1]$, such that $\partial P=T \cup \partial C_{0}$ 
and $P \cap C_{0}=\partial C_{0}$. Since $C_{0}$ is diffeomorphic to $T^{2} \times[0, \infty)$, the submanifold $C:=C_{0} \cup P$, which is closed as a subset of $M$ and has boundary $T$, is also diffeomorphic to $T^{2} \times[0, \infty)$. Furthermore, since $C_{0}$ has finite volume and $P$ is compact, $C$ also has finite volume.

To prove (3), consider a torus $T \subset M$ which is not incompressible. In this case, by [15, Lemma 6.1], there is a disk $D \subset \operatorname{int} M$ such that $D \cap T=\partial D$, and $\partial D$ is a non-trivial, and hence non-separating, curve on $T$. Let $E$ be a 3 -ball containing $D$, such that $A:=E \cap T$ is an annular neighborhood of $\partial D$ in $T$ and $A \subset \partial E$. Then $S:=(T \cup \partial E)-\operatorname{int} A$ is a 2 -sphere which must bound a 3 -ball $B \subset M$, since $M$ is irreducible by (1). We must have either $E \subset B$ or $E \cap B=\overline{(\partial E)-A}$. In the first case we have $T \subset B$, and (ii) holds. In the second case, $J:=E \cup B$ is a solid torus since $M$ is orientable, and $\partial J=T$, so that (b) holds.

The following well-known consequence of the sphere theorem is included, for example, in [11, Theorem 8.2]:

Proposition 3.6. An irreducible 3-manifold with infinite fundamental group is aspherical.

The following two facts about 3-manifolds are well known, but we have supplied proofs for completeness.

Proposition 3.7. Let $N$ be a compact, irreducible, orientable 3-manifold such that every component of $\partial N$ is a torus. If $N$ is boundary-reducible, then it is a solid torus.

Proof. We will prove the corresponding statement in the PL category (see Section 3.1). Let $N$ be a compact, irreducible, orientable PL 3-manifold such that every component of $\partial N$ is a torus. If $N$ is boundary-reducible, it contains an essential disk $D$. By hypothesis the component of $\partial N$ containing $\partial D$ is a torus $T$. If $E$ denotes a regular neighborhood of $D$ in $N$, then the component of $\partial(\overline{N-E})$ that meets $T$ is a 2-sphere. By irreducibility it follows that $\overline{N-E}$ is a ball, so that $N$ is a solid torus.

Proposition 3.8. Suppose that $M$ is an irreducible, orientable 3-manifold. Then there is a compact, irreducible submanifold $N$ of $M$ such that the inclusion homomorphism $\pi_{1}(N) \rightarrow \pi_{1}(M)$ is an isomorphism.

Proof. According to [30, there is a compact submanifold $N_{0}$ of $M$ such that the inclusion homomorphism $\pi_{1}\left(N_{0}\right) \rightarrow \pi_{1}(M)$ is an isomorphism. By Lemma 3.1. there is a compact irreducible submanifold $N$ of $M$ such that $N \supset N_{0}$, and the inclusion homomorphism $\pi_{1}\left(N_{0}\right) \rightarrow \pi_{1}(N)$ is surjective. It follows that the inclusion homomorphism $\pi_{1}(N) \rightarrow \pi_{1}(M)$ is an isomorphism.

We will make use of the characteristic submanifold theory [17, 18]. The information that we will need is summarized in the following statement:

Proposition 3.9. Let $M$ be any Haken manifold. Then there is a Seifert-fibered manifold $\Sigma \subset$ int $M$ such that each component of $\partial \Sigma$ is incompressible in $M$ and has the following property: if $f: T^{2} \rightarrow M$ is any map such that $f_{\sharp}: \pi_{1}\left(T^{2}\right) \rightarrow \pi_{1}(M)$ is injective, then $f$ is homotopic to a map $g: T^{2} \rightarrow \operatorname{int} M$ such that $g\left(T^{2}\right) \subset \Sigma$. 
Proof. According to the statement of the Characteristic Pair Theorem on page 138 of [17] and the discussion of the case $T=\emptyset$ following that statement, there is a Seifert-fibered manifold $\Sigma \subset \operatorname{int} M$ such that each component of $\partial \Sigma$ is incompressible in $M$ and has the following property: if $\mathcal{S}$ is any Seifert fibered space and $F: \mathcal{S} \rightarrow M$ is any map which is non-degenerate (in the sense defined on p. 55 of [17, taking $\mathcal{F}=\mathcal{T}=\emptyset$ ), then $F$ is homotopic to a map whose image is contained in $\Sigma$. Now if $f: T^{2} \rightarrow M$ is any map such that $f_{\sharp}: \pi_{1}\left(T^{2}\right) \rightarrow \pi_{1}(M)$ is injective and if we let $q: T^{2} \times[0,1] \rightarrow T^{2}$ denote the projection to the first factor, it follows immediately from the definition that $f \circ q$ is a non-degenerate map of the Seifert fibered manifold $T^{2} \times[0,1]$ into $M$. Hence $f \circ q$ is homotopic to a map of $T^{2} \times[0,1]$ into $M$ whose image is contained in $\Sigma$, and so $f$ is homotopic to a map $g: T^{2} \rightarrow \operatorname{int} M$ such that $g\left(T^{2}\right) \subset \Sigma$.

(It may be shown that the submanifold $\Sigma$ given by Proposition 3.9 is unique up to ambient isotopy in $M$, but we will not need this fact. Note that although $\Sigma \subset$ int $Q$, every torus component $T$ of $\partial Q$ which is $\pi_{1}$-injective in $M$ is "parallel" to a component of $\partial \Sigma$. One may think of $\Sigma$ as an "absolute" characteristic submanifold of $Q$ which "carries essential singular tori", as distinguished from the "relative" characteristic submanifold which is defined only when $Q$ is boundary-irreducible, and carries both essential singular tori and essential singular annuli.)

The next two results, Propositions 3.10 and 3.11, are closely related to Theorem VI.4.1 of [17. They are both essentially topological results, although we have found it more convenient to state Proposition 3.11 in the setting of hyperbolic 3-manifolds.

Proposition 3.10. Let $N$ be a compact, irreducible, orientable 3-manifold such that $\pi_{1}(N)$ has rank 2 and is not free. Then each component of $\partial N$ is a torus.

Proof. If $N$ is closed, there is nothing to prove. If $\partial N \neq \emptyset$, then $N$ has the homotopy type of a connected finite CW complex $K$ of dimension at most 2 . We may take $K$ to have only one 0 -cell. Let $m$ and $n$ denote, respectively, the numbers of 1-cells and 2-cells of $K$. Then $\pi_{1}(K)$ has a presentation with $m$ generators and $n$ relations. By definition, the deficiency of this presentation is $m-n$. It is shown in 22] that if $k$ is a positive integer, a finitely presented group that has rank $k$ and has a presentation of deficiency at least $k$ must be free. Since $\pi_{1}(K) \cong \pi_{1}(N)$ has rank 2 and is not free, the deficiency $m-n$ must be at most one. This gives

$$
\frac{1}{2} \chi(\partial N)=\chi(N)=\chi(K)=1-m+n \geq 0 .
$$

Note that if some component of $\partial N$ were a sphere, then by irreducibility $N$ would be a ball, which is impossible since $\pi_{1}(N)$ has rank 2 . Thus $\partial N$ is a closed, orientable 2 -manifold with no sphere components, and $\chi(N) \geq 0$. Hence every component of $\partial N$ is a torus.

Proposition 3.11. Let $M$ be a hyperbolic 3-manifold of infinite volume. Then every two-generator non-abelian subgroup of $\pi_{1}(M)$ is free.

Proof. Let $X \leq \pi_{1}(M)$ be a two-generator non-abelian subgroup. Then $X \cong$ $\pi_{1}(\widetilde{M})$ for some covering space $\widetilde{M}$ of $M$. Since $M$ is irreducible by assertion (1) of Proposition 3.5] it follows from [23, p. 647, Theorem 3] that $\widetilde{M}$ is irreducible. By Proposition 3.8 there is a compact, irreducible submanifold $N$ of $M$ such that the inclusion homomorphism $\pi_{1}(N) \rightarrow \pi_{1}(M)$ is an isomorphism. If $X \cong \pi_{1}(N)$ is not free, then it follows from Proposition 3.10 that every component of $\partial M$ is a 
torus. Since $\pi_{1}(N) \cong X$ is non-abelian by hypothesis, $N$ is not a solid torus; hence by Proposition 3.7. $N$ is boundary-irreducible. If $T$ is any component of $\partial N$, it follows that $T$ is $\pi_{1}$-injective in $N$, and therefore in $M$ as well. Hence by assertion (2) of Proposition 3.5 $T$ is the boundary of a submanifold $C_{T}$ of $M$ which is closed as a subset of $M$, is diffeomorphic to $T^{2} \times[0, \infty)$, and has finite volume. Since $\pi_{1}(N)$ is non-abelian and since the inclusion homomorphism $\pi_{1}(N) \rightarrow \pi_{1}(M)$ is in particular injective, we cannot have $C_{T} \supset N$ for any $T$. Hence we must have $C_{T} \cap N=T$ for each $T$ so that $M=N \cup \bigcup_{T} C_{T}$. But this implies that $M$ has finite volume, a contradiction.

The following result has direct relevance to estimating Margulis numbers, and its corollaries were mentioned in the introduction.

Proposition 3.12. Let $x$ and $y$ be non-commuting elements of $\operatorname{Isom}_{+}\left(\mathbb{H}^{3}\right)$ such that $\langle x, y\rangle$ is discrete and torsion-free and has infinite covolume. Then for every $P \in \mathbb{H}^{3}$ we have

$$
\max (d(P, x \cdot P), d(P, y \cdot P)) \geq \log 3 .
$$

Proof. Set $\Gamma=\langle x, y\rangle$. Proposition 3.11, applied to the hyperbolic 3-manifold $M=$ $\mathbb{H}^{3} / \Gamma$, shows that $\Gamma$ is free. The conclusion now follows from the case $k=2$ of [2, Theorem 4.1].

Corollary 3.13. Let $M$ be an orientable hyperbolic 3-manifold such that every subgroup of rank at most 2 in $\pi_{1}(M)$ has infinite index. Then $\mu(M) \geq \log 3=$ $1.09 \ldots$

Proof. Write $M=\mathbb{H}^{3} / \Gamma$, where $\Gamma \leq \operatorname{Isom}_{+}\left(\mathbb{H}^{3}\right)$ is discrete and torsion-free. Let $x$ and $y$ be non-commuting elements of $\Gamma$. The hypothesis implies that $\langle x, y\rangle$ has infinite index in $\Gamma$, and hence has infinite covolume. By Proposition 3.12 , it follows that $\max (d(P, x \cdot P), d(P, y \cdot P)) \geq \log 3$ for every $P \in \mathbb{H}^{3}$. Hence $\log 3$ is a Margulis number for $M$.

Corollary 3.14. Let $M$ be an orientable hyperbolic 3-manifold such that either $H_{1}(M ; \mathbb{Q})$ has rank at least 3 or $M$ is closed and $H_{1}\left(M ; \mathbb{Z}_{p}\right)$ has rank at least 4 for some prime $p$. Then $\mu(M) \geq \log 3$.

Proof. If $H_{1}(M ; \mathbb{Q})$ has rank at least 3 , it is clear that every subgroup of rank at most 2 in $\pi_{1}(M)$ has infinite index. If $M$ is closed and $H_{1}\left(M ; \mathbb{Z}_{p}\right)$ has rank at least 4 for some prime $p$, then by [34, Proposition 1.1], it is again true that every subgroup of rank at most 2 in $\pi_{1}(M)$ has infinite index. Hence the result follows from Corollary 3.13 .

4. An AbStract Bound FOR vol $M$ WHen $\mu(M)<\log 3$, AND ITS CONSEQUENCES

Let $\left(\rho_{n}\right)_{n \geq 1}$ be a sequence of representations of a group $X$ in $\operatorname{Isom}_{+}\left(\mathbb{H}^{3}\right)$. Recall that the sequence $\left(\rho_{n}\right)$ is said to converge algebraically to a representation $\rho_{\infty}$ of $X$ in $\operatorname{Isom}_{+}\left(\mathbb{H}^{3}\right)$ if we have $\rho_{n}(\gamma) \rightarrow \rho_{\infty}(\gamma)$ for every $\gamma \in X$.

Recall that a subgroup of $\operatorname{Isom}_{+}\left(\mathbb{H}^{3}\right)$ is said to be elementary if it has an abelian subgroup of finite index. According to [32, Proposition 2.1], every torsion-free, elementary, discrete subgroup of $\operatorname{Isom}_{+}\left(\mathbb{H}^{3}\right)$ is abelian.

We will give an explicit proof of the following fact, which is implicit in 20. 
Lemma 4.1. Let $\left(\rho_{n}\right)_{n \geq 1}$ be a sequence of representations of a finitely generated group $\Phi$ in $\operatorname{Isom}_{+}\left(\mathbb{H}^{3}\right)$. Suppose that $\rho_{n}(\Phi)$ is discrete, non-elementary and torsionfree for each $n \in \mathbb{N}$. Then there is a neighborhood $W$ of the identity in $\operatorname{Isom}_{+}\left(\mathbb{H}^{3}\right)$ such that $\widetilde{\Gamma}_{n} \cap W=\{1\}$ for every $n \in \mathbb{N}$.

Proof. If the conclusion is false, there is a sequence of elements $\left(x_{n}\right)_{n \geq 1}$ of $\Phi$ such that $\rho_{n}\left(x_{n}\right) \neq 1$ for $n=1,2, \ldots$ but $\rho_{n}\left(x_{n}\right) \rightarrow \infty$ as $n \rightarrow \infty$. Since $\rho_{n}(\Phi)$ is a non-abelian, torsion-free, discrete subgroup of $\mathrm{Isom}_{+}$, it has trivial center. In particular, $\rho_{n}\left(x_{n}\right) \neq 1$ is non-central in $\rho_{n}(\Phi)$ for each $n \in \mathbb{N}$. Hence if $S$ is a finite generating set for $\Phi$, then for each $n \in \mathbb{N}$ there is an element $s_{n} \in S$ such that $\rho_{n}\left(x_{n}\right)$ and $\rho_{n}\left(s_{n}\right)$ do not commute. Since $S$ is finite, we may assume after passing to a subsequence that the $s_{n}$ are all the same element of $S$, say $s$.

For each $n \in \mathbb{N}$, the group $\Gamma_{n}:=\left\langle\rho_{n}\left(x_{n}\right), \rho_{n}(s)\right\rangle$ is contained in $\rho_{n}(\Phi)$ and is therefore discrete and torsion-free. Since $\rho_{n}\left(x_{n}\right)$ and $\rho_{n}(s)$ do not commute, $\Gamma_{n}$ is non-abelian and is therefore non-elementary by [32, Proposition 2.1]. If $X_{n}$ and $Y_{n}$ are elements of $\mathrm{SL}_{2}(\mathbb{C})$ representing $\rho_{n}\left(x_{n}\right)$ and $\rho_{n}(s)$ respectively, it then follows from Jorgensen's inequality [20, Lemma 1] that

$$
\left|\left(\operatorname{trace} X_{n}\right)^{2}-4\right|+\left|\operatorname{trace}\left(X_{n} Y_{n} X_{n}^{-1} Y_{n}^{-1}\right)-2\right| \geq 1
$$

for each $n \in \mathbb{N}$. As $n \rightarrow \infty$ we have $\rho_{n}\left(x_{n}\right) \rightarrow 1$, while $\rho_{n}(s) \rightarrow \rho_{\infty}(s)$, where $\rho_{\infty}$ denotes the algebraic limit of the sequence $\left(\rho_{n}\right)$. After passing to a subsequence we may therefore assume that $X_{n} \rightarrow \pm I$ and that the sequence $\left(Y_{n}\right)$ has a limit. Hence $X_{n} Y_{n} X_{n}^{-1} Y_{n} \rightarrow I$. It follows that the left hand side of (4.0.1) converges to 0 as $n \geq \infty$, a contradiction.

The following result was stated in the introduction as Theorem A.

Theorem 4.2. Let $\lambda$ be a positive real number strictly less than $\log 3$. Then there is a constant $V_{\lambda}$ such that for every orientable hyperbolic 3-manifold $M$ with $\mu(M)<\lambda$ we have $\operatorname{vol} M \leq V_{\lambda}$ (and in particular vol $M<\infty$ ).

Proof. It follows immediately from Proposition 3.12 that if $M$ is any orientable 3manifold of infinite volume, then $\log 3$ is a Margulis number for $M$, so that $\mu(M) \geq$ $\log 3>\lambda$. Hence it suffices to show that there is a constant $V_{\lambda}$ such that for every orientable hyperbolic 3-manifold $M$ with $\infty>\operatorname{vol} M>V_{\lambda}$, we have $\mu(M) \geq \lambda$.

We reason by contradiction. Assume there is a sequence $\left(M_{n}\right)_{n \geq 1}$ of orientable finite-volume hyperbolic 3-manifolds such that vol $M_{n} \rightarrow \infty$ and $\mu\left(M_{n}\right)<\lambda$, i.e., no $M_{n}$ admits $\lambda$ as a Margulis number.

For each $n$ write $M_{n}=\mathbb{H}^{3} / \Gamma_{n}$ for some torsion-free cocompact discrete subgroup $\Gamma_{n}$ of $\operatorname{Isom}_{+}\left(\mathbb{H}^{3}\right)$. Then, by definition, for each $n$ there exist non-commuting elements $x_{n}, y_{n} \in \Gamma_{n}$ and a point $P_{n} \in \mathbb{H}^{3}$ such that

$$
\max \left(d\left(P_{n}, x_{n} \cdot P_{n}\right), d\left(P_{n}, y_{n} \cdot P_{n}\right)\right)<\lambda .
$$

After replacing each $\Gamma_{n}$ by a suitable conjugate of itself in $\operatorname{Isom}_{+}\left(\mathbb{H}^{3}\right)$, we may assume that the $P_{n}$ are all the same point of $\mathbb{H}^{3}$, which we will denote by $P$. Thus for each $n$ we have

$$
\max \left(d\left(P, x_{n} \cdot P\right), d\left(P, y_{n} \cdot P\right)\right)<\lambda .
$$

For each $n$, set $\widetilde{\Gamma}_{n}:=\left\langle x_{n}, y_{n}\right\rangle$ and $\widetilde{M}_{n}:=\mathbb{H}^{3} / \widetilde{\Gamma}_{n}$. Note that $\widetilde{\Gamma}_{n}$ is discrete and torsion-free since $\Gamma_{n}$ is, and that $\widetilde{\Gamma}_{n}$ is non-abelian - and hence non-elementary by [32, Proposition 2.1] - since $x_{n}$ and $y_{n}$ do not commute. 
Since $\lambda<\log 3$, it follows from (4.0.2) and Proposition 3.12 that vol $\widetilde{M}_{n}<\infty$. On the other hand, $\widetilde{M}_{n}$ covers $M_{n}$, and hence vol $\widetilde{M}_{n} \geq \operatorname{vol} M_{n}$. In particular, $\operatorname{vol} \widetilde{M}_{n} \rightarrow \infty$.

It follows from (4.0.2) that the $x_{n}$ and $y_{n}$ lie in a compact subset of $\operatorname{Isom}_{+}\left(\mathbb{H}^{3}\right)$. Hence, after passing to a subsequence, we may assume that the sequences $\left(x_{n}\right)$ and $\left(y_{n}\right)$ converge in $\operatorname{Isom}_{+}\left(\mathbb{H}^{3}\right)$ to limits $x_{\infty}$ and $y_{\infty}$. Again by (4.0.2), we have

$$
\max \left(d\left(P, x_{\infty} \cdot P\right), d\left(P, y_{\infty} \cdot P\right)\right) \leq \lambda .
$$

For $1 \leq n \leq \infty$ we define a representation $\rho_{n}$ of the rank-2 free group $F_{2}=\langle\xi, \eta\rangle$ by $\rho_{n}(\xi)=x_{n}, \rho_{n}(\eta)=y_{n}$. Thus $\rho_{n}\left(F_{2}\right)=\widetilde{\Gamma}_{n}$ for each $n$. Since $\xi$ and $\eta$ generate $F_{2}$ and since $\rho_{n}(\xi) \rightarrow \rho_{\infty}(\xi)$ and $\rho_{n}(\eta) \rightarrow \rho_{\infty}(\eta)$ as $n \rightarrow \infty$, we have $\rho_{n}(\gamma) \rightarrow$ $\rho_{\infty}(\gamma)$ for every $\gamma \in F_{2}$. By definition this means that the sequence $\left(\rho_{n}\right)$ converges algebraically to $\rho_{\infty}$. Set $x_{\infty}=\rho_{\infty}(\xi), y_{\infty}=\rho_{\infty}(\eta)$.

Let $D$ denote the set of representations of $F_{2}$ in $\operatorname{Isom}_{+}\left(\mathbb{H}^{3}\right)$ whose images are discrete, torsion-free, and non-elementary. According to [32, Theorem 2.4] (a theorem essentially due to T. Jorgensen and P. Klein [21]), the limit of any algebraically convergent sequence of representations in $D$ is again in $D$. Hence $\rho_{\infty} \in D$. Thus $\widetilde{\Gamma}_{\infty}:=\rho_{\infty}\left(F_{2}\right)=\left\langle x_{\infty}, y_{\infty}\right\rangle$ is a discrete group.

According to [19, Proposition 3.8], since $\left(\rho_{n}\right)$ converges algebraically, the sequence of discrete groups $\left(\widetilde{\Gamma}_{n}\right)$ has a geometrically convergent subsequence (in the sense defined in [19]). Hence without loss of generality we may assume that $\left(\widetilde{\Gamma}_{n}\right)$ converges geometrically to some discrete group $\widehat{\Gamma}_{\infty}$. It then follows, again from 19 , Proposition 3.8], that $\widetilde{\Gamma}_{\infty} \leq \widehat{\Gamma}_{\infty}$.

According to Lemma 4.1, there is a neighborhood $W$ of the identity in $\operatorname{Isom}_{+}\left(\mathbb{H}^{3}\right)$ such that $\widetilde{\Gamma}_{n} \cap W=\{1\}$ for every $n \in \mathbb{N}$. Let $E$ denote the set of all torsion-free subgroups $\Delta$ of $\operatorname{Isom}_{+}\left(\mathbb{H}^{3}\right)$ such that $\Delta \cap W=\{1\}$. (In particular, each group in $E$ is discrete.) According to [7, Theorem 1.3.1.4], $E$ is compact in the topology of geometric convergence. Since $\widetilde{\Gamma}_{n} \in E$ for each $n \in \mathbb{N}$, we have $\widehat{\Gamma}_{\infty} \in E$. In particular, $\widehat{\Gamma}_{\infty}$ is torsion-free. We let $\widehat{M}_{\infty}$ denote the orientable hyperbolic 3manifold $\mathbb{H}^{3} / \widehat{\Gamma}_{\infty}$.

Since $\left(\widetilde{\Gamma}_{n}\right)$ converges geometrically to $\widehat{\Gamma}_{\infty}$, the sequence of orientable hyperbolic 3-manifolds $\left(\widetilde{M}_{n}\right)$ converges geometrically to $\widehat{M}_{\infty}$ in the sense of [5. Chapter E]. If vol $\widehat{M}_{\infty}$ were finite, it would then follow from [5. Proposition E.2.5] that the sequence $\left(\operatorname{vol} \widetilde{M}_{n}\right.$ ) had the finite limit vol $\widehat{M}_{\infty}$, which contradicts vol $\widetilde{M}_{n} \rightarrow \infty$. Thus $\widetilde{M}_{\infty}:=\mathbb{H}^{3} / \rho_{\infty}\left(F_{2}\right)$ is a hyperbolic 3 -manifold of infinite volume. It therefore follows from Proposition 3.12 that

$$
\max \left(d\left(P, x_{\infty} \cdot P\right), d\left(P, y_{\infty} \cdot P\right)\right) \geq \log 3 .
$$

But this contradicts 4.0.3).

The following two corollaries of Theorem 4.2 were also pointed out in the introduction.

Corollary 4.1. Let $\lambda$ be a positive real number strictly less than $\log 3$. Then there is a natural number $d_{\lambda}$ such that for every orientable hyperbolic 3-manifold $M$ with $\mu(M)<\lambda$, the group $\pi_{1}(M)$ has a rank-2 subgroup of index at most $d_{\lambda}$.

Proof. Let $v$ denote the infimum of the volumes of all hyperbolic 3-manifolds; we have $v>0$ (for example by [24, Theorem 1]). Let $V_{\lambda}$ be a positive real number 
having the property stated in Theorem 4.2, and set

$$
d_{\lambda}=\left\lfloor\frac{V_{\lambda}}{v}\right\rfloor .
$$

Suppose that $M$ is an orientable hyperbolic 3-manifold such that $\mu(M)<\lambda$, i.e., such that $\lambda$ is not a Margulis number for $M$. Write $M_{\infty}=\mathbb{H}^{3} / \Gamma$, where $\Gamma \leq \operatorname{Isom}_{+}\left(\mathbb{H}^{3}\right)$ is discrete and torsion-free. Then by definition there exist a point $P \in \mathbb{H}^{3}$ and non-commuting elements $x, y \in \Gamma$ such that

$$
\max (d(P, x \cdot P), d(P, y \cdot P))<\lambda .
$$

Set $\widetilde{\Gamma}=\langle x, y\rangle$ and $\widetilde{M}=\mathbb{H}^{3} / \widetilde{\Gamma}$. Since $x$ and $y$ are non-commuting elements of $\widetilde{\Gamma}$, it follows from (4.1.1) that $\lambda$ is not a Margulis number for $\widetilde{M}$, i.e., that $\mu(M)<\lambda$. Hence vol $\widetilde{M} \leq V_{\lambda}<\infty$, and since $\widetilde{M}$ covers $M$ we have vol $M<\infty$. Since $\operatorname{vol} M \geq v$, we find that

$$
[\Gamma: \widetilde{\Gamma}]=\frac{\operatorname{vol} \widetilde{M}}{\operatorname{vol} M} \leq \frac{V_{\lambda}}{v} .
$$

It follows that $[\Gamma: \widetilde{\Gamma}] \leq d_{\lambda}$, so that $\Gamma \cong \pi_{1}(M)$ has a rank-2 subgroup of index at most $d_{\lambda}$.

Corollary 4.2. Let $\lambda$ be a positive real number strictly less than $\log 3$. Then there is a natural number $k_{\lambda}$ such that for every orientable hyperbolic 3-manifold $M$ with $\mu(M)<\lambda$, the group $\pi_{1}(M)$ has rank at most $k_{\lambda}$.

Proof. Let $d_{\lambda}$ be a natural number having the property stated in Corollary 4.1. Set $k_{\lambda}=2+\left\lfloor\log _{2} d_{\lambda}\right\rfloor$. If $M$ is an orientable hyperbolic 3-manifold with $\mu(M)<\lambda$, then the group $\Gamma=\pi_{1}(M)$ has a rank-2 subgroup $\widetilde{\Gamma}$ of index at most $d_{\lambda}$. According to Proposition 2.1, we have

$$
\operatorname{rank} \Gamma \leq \operatorname{rank} \widetilde{\Gamma}+\log _{2}[\Gamma: \widetilde{\Gamma}] \leq 2+d_{\lambda},
$$

and hence $\pi_{1}(M) \cong \Gamma$ has rank at most $k_{\lambda}$.

\section{MARgulis Numbers AND SHORT RELATIONS}

Notation 5.1 . Let $\lambda$ be a real number $0<\lambda<(\log 3) / 2$. Then for any sufficiently large positive integer $N$ we have

$$
\frac{3^{N}-1}{4 N+1} \geq 2667(\sinh (2 N \lambda+.104)-(2 N \lambda+.104)) .
$$

(The natural logarithm of the left hand side of (5.1.1) is asymptotic to $N \log 3$, whereas the natural logarithm of the right hand side is asymptotic to $2 N \lambda>$ $N \log 3$.

We shall denote by $N(\lambda)$ the smallest positive integer $N$ for which (5.1.1) holds.

Here is one simple estimate of $N(\lambda)$ :

Proposition 5.2. Let $\lambda \in(0.1,(\log 3) / 2)$ be given, and set $\beta=1 /((\log 3)-2 \lambda)$. Then

$$
N(\lambda)<1+110 \beta \log \beta
$$


Proof. Since $\lambda>0.1$ we have $\beta>1 /((\log 3)-0.2)>1.11$. Hence the right hand side of (5.2.1) is bounded below by $1+(110)(1.11)(\log 1.11)=13.7 \ldots$. We may therefore assume without loss of generality that $N(\lambda) \geq 14$.

Set $n=N(\lambda)-1 \geq 13$. From the definition of $N(\lambda)$ we have

$$
\frac{3^{n}-1}{4 n+1}<2667(\sinh (2 n \lambda+.104)-(2 n \lambda+.104)) .
$$

Since in particular we have $n \geq 3$, the left hand side of $(5.2 .2)$ is bounded below by $3^{n} / 5 n$. The right hand side is obviously bounded above by $2667 \exp (2 n \lambda+.104) / 2$. Hence

$$
3^{n} \leq \frac{2667}{2} \cdot 5 n \cdot \exp (2 n \lambda+.104)<7400 n e^{2 n \lambda},
$$

which upon taking logarithms and using the definition of $\beta$ gives

$$
n<\beta \log (7400 n) \text {. }
$$

Now suppose that (5.2.1) is false, so that $n \geq 110 \beta \log \beta$. Define a function $g(x)$ for $x>0$ by $g(x)=x / \log (7400 x)$. Then $g(x)$ is monotone increasing for $x>e / 7400$, and since $n \geq 110 \beta \log \beta>12.7$, we have $g(n) \geq g(110 \beta \log \beta)$. With (5.2.3) this gives

so that

$$
\frac{110 \beta \log \beta}{\log (7400 \cdot 110 \beta \log \beta)} \leq \frac{n}{\log (7400 n)}<\beta,
$$

$110 \log \beta<\log 7400+\log 110+\log \beta+\log \log \beta<13.61+\log \beta+\log \log \beta$, i.e.,

$$
109 \log \beta-\log \log \beta<13.61 .
$$

On the other hand, the function $h(x):=109 x-\log x$ is monotonically increasing for $x>1 / 109$. Since $\log \beta>\log 1.11=0.104 \ldots>1 / 109$, we have

$$
109 \log \beta-\log \log \beta=h(\log \beta)>h(\log 1.11)=13.63 \ldots,
$$

which contradicts (5.2.4).

Proposition 5.3. Let $M$ be an orientable hyperbolic 3-manifold and write $M=$ $\mathbb{H}^{3} / \Gamma$, where $\Gamma \leq \operatorname{Isom}_{+}\left(\mathbb{H}^{3}\right)$ is discrete and torsion-free. Let $\lambda<(\log 3) / 2$ be given, and let $x$ and $y$ be non-commuting elements of $\Gamma$ such that

$$
\max (d(P, x \cdot P), d(P, y \cdot P))<\lambda .
$$

Then there is a reduced word $W$ in two letters, with $0<$ length $W \leq 8 N(\lambda)$, such that $W(x, y)=1$. (Here $N(\lambda)$ is defined by [5.1])

Proof. Set $\mu=0.104$. We pointed out in the introduction that, according to 24, we have $\mu<\mu_{+}(3)$; that is, $\mu$ is a Margulis number for every orientable hyperbolic 3 -manifold. Set $N=N(\lambda)$.

Since $\mu$ is in particular a Margulis number for $M$, the elements $\gamma \in \Gamma$ such that $d(\gamma \cdot P, P)<\mu$ generate an abelian subgroup $C$ of $\Gamma$.

Let $F_{2}$ denote a free group on two (abstract) generators $\xi$ and $\eta$. We identify $F_{2}$ with the set of all reduced words in $\xi$ and $\eta$, so that $V(\xi, \eta)=V$ for every reduced word $V$. Let $\phi: F_{2} \rightarrow \Gamma$ denote the unique homomorphism such that $\phi(\xi)=x$ and $\phi(\eta)=y$. Then $\phi(V)=V(x, y)$ for every reduced word $V$.

For every positive integer $n$, let $\mathcal{V}_{n} \subset F_{2}$ denote the set of all reduced words of length at most $n$ in $\xi$ and $\eta$. If $m$ and $n$ are positive integers, then for all $V \in \mathcal{V}_{m}$ 
and $V^{\prime} \in \mathcal{V}_{n}$, we can concatenate $V$ and $V^{\prime}$ and then reduce the resulting word to obtain a reduced word of length at most $m+n$ representing the product $V V^{\prime}$ in $F_{2}$. Hence

$$
\mathcal{V}_{m} \mathcal{V}_{n} \subset \mathcal{V}_{m+n} \text { for all } m, n>0
$$

Also note that

$$
\mathcal{V}_{n}^{-1}=\mathcal{V}_{n} \text { for every } n>0 .
$$

For any $k>0$, the number of reduced words in $\xi$ and $\eta$ of length exactly $k$ is $4 \cdot 3^{k-1}$. Summing from $k=1$ to $k=n$, we deduce that

$$
\#\left(\mathcal{V}_{n}\right)-1=2\left(3^{n}-1\right) \text { for every } n \geq 1 \text {. }
$$

We will assume that

$$
\phi(W) \neq 1 \text { for every } W \in \mathcal{V}_{8 N}-\{1\} .
$$

Under the assumption of (5.3.4) we will derive a contradiction, thereby proving the proposition.

Assuming (5.3.4), we claim that

$$
\#\left(\mathcal{V}_{N} \cap \phi^{-1}(\gamma C)\right) \leq 4 N+1 \text { for any left coset } \gamma C \text { of } C \text { in } \Gamma \text {. }
$$

To prove (5.3.5) we will first consider the special case in which $\phi^{-1}(C) \cap \mathcal{V}_{2 N}=$ $\{1\}$. In this case, if $V$ and $V^{\prime}$ are elements of $\mathcal{V}_{N} \cap \phi^{-1}(\gamma C)$ for a given left coset $\gamma C$ of $C$ in $\Gamma$, we have $U:=V^{-1} V^{\prime} \in \phi^{-1}(C)$; but by (5.3.1) and (5.3.2) we have $U \in \mathcal{V}_{2 N}$. The assumption $\phi^{-1}(C) \cap \mathcal{V}_{2 N}=\{1\}$ then implies that $U=1$ and hence that $V=V^{\prime}$. Hence in this case we have $\#\left(\mathcal{V}_{N} \cap \phi^{-1}(\gamma C)\right) \leq 1$, which is stronger than (5.3.5).

Hence in proving (5.3.5) we may assume that $\phi^{-1}(C) \cap \mathcal{V}_{2 N} \neq\{1\}$. Of course we may also assume that $\mathcal{V}_{N} \cap \phi^{-1}(\gamma C) \neq \emptyset$. Let us fix an element $U_{0} \neq 1$ of $\phi^{-1}(C) \cap \mathcal{V}_{2 N}$ and an element $V_{1}$ of $\mathcal{V}_{N} \cap \phi^{-1}(\gamma C)$. Let $\widehat{C}$ denote the centralizer of $U_{0}$ in $F_{2}$; then $\widehat{C}$ is cyclic, since $F_{2}$ is free and $U_{0} \neq 1$.

Let us define an injective map $J: \mathcal{V}_{N} \cap \phi^{-1}(\gamma C) \rightarrow F_{2}$ by $J(V)=V^{-1} V_{1}$. Since $V_{1} \in \phi^{-1}(\gamma C)$, we have $J(V) \in \phi^{-1}(C)$ for every $V \in \mathcal{V}_{N} \cap \phi^{-1}(\gamma C)$. Since $V_{1} \in \mathcal{V}_{N}$, it follows from (5.3.1) and (5.3.2) that $J(V) \in \mathcal{V}_{2 N}$ for every $V \in \mathcal{V}_{N} \cap \phi^{-1}(\gamma C)$. Thus $J$ maps $\mathcal{V}_{N} \cap \phi^{-1}(\gamma C)$ into $\mathcal{V}_{2 N} \cap \phi^{-1}(C)$.

Let $V \in \mathcal{V}_{N} \cap \phi^{-1}(\gamma C)$ be given. Since $U_{0}, J(V) \in \phi^{-1}(C)$, we have

$$
\phi\left(J(V) U_{0} J(V)^{-1} U_{0}^{-1}\right)=1 .
$$

Since $J(V)$ and $U_{0}$ belong to $\mathcal{V}_{2 N}$, it follows from (5.3.1) and (5.3.2) that

$$
J(V) U_{0} J(V)^{-1} U_{0}^{-1} \in \mathcal{V}_{8 N} .
$$

Applying (5.3.4) with $W=J(V) U_{0} J(V)^{-1} U_{0}^{-1}$ we deduce that $W=1$, i.e., that $J(V)$ commutes with $U_{0}$. This means that $J(V) \in \hat{C}$.

Thus $J$ is an injection from $\mathcal{V}_{N} \cap \phi^{-1}(\gamma C)$ to $\mathcal{V}_{2 N} \cap \widehat{C}$, and so $\#\left(\mathcal{V}_{N} \cap \phi^{-1}(\gamma C)\right) \leq$ $\#\left(\mathcal{V}_{2 N} \cap \widehat{C}\right)$. But according to Lemma 2.1 applied with $k=2 N$ and with $Z=\widehat{C}$, we have $\#\left(\mathcal{V}_{2 N} \cap \widehat{C}\right) \leq 4 N+1$. Thus (5.3.5) is established.

Now let $\mathcal{L}$ denote the set of all left cosets of $C$ in $\Gamma$, and define a map $\psi: F_{2} \rightarrow \mathcal{L}$ by $\psi(V)=\phi(V) C$. We may then paraphrase (5.3.5) by saying that the fibers of the surjection $\psi \mid \mathcal{V}_{N}: \mathcal{V}_{N} \rightarrow \psi\left(\mathcal{V}_{N}\right)$ have cardinality at most $4 N+1$. If we set 
$r=\#\left(\psi\left(\mathcal{V}_{N}\right)\right)$, it follows that $r \geq \#\left(\mathcal{V}_{N}\right) /(4 N+1)$. Combining this with (5.3.3), we find that

$$
r>\frac{2\left(3^{N}-1\right)}{4 N+1}
$$

Let $b$ denote the open ball of radius $\mu / 2$ centered at $P$, and let $B$ denote the ball of radius $N \lambda+(\mu / 2)$ centered at $P$. We have

$$
\operatorname{vol} b=\pi(\sinh (\mu)-\mu)=0.000589 \ldots
$$

and

$$
\operatorname{vol} B=\pi(\sinh (2 N \lambda+\mu)-(2 N \lambda+\mu)) .
$$

Since $d(x \cdot P, P)<\lambda$ and $d(y \cdot P, P)<\lambda$, and since $x$ and $y$ are isometries, we have $d(\phi(V) \cdot P, P)=d(V(x, y) \cdot P, P)<N \lambda$ for every $V \in \mathcal{V}_{N}$. It follows that

$$
\phi(V) \cdot b \subset B \text { for every } V \in \mathcal{V}_{N} .
$$

According to the definition of $r$, there are elements $\gamma_{1}, \ldots, \gamma_{r}$ of $\phi\left(\mathcal{V}_{N}\right)$ which represent distinct left cosets of $C$ in $\gamma$. From (5.3.9) we have

$$
\gamma_{i} \cdot b \subset B \text { for } i=1, \ldots, r .
$$

If $i$ and $j$ are distinct indices in $\{1, \ldots, r\}$ we have $\gamma_{j}^{-1} \gamma_{i} \notin C$, which by the definition of $C$ gives $d\left(\gamma_{i} \cdot P, \gamma_{j} \cdot P, P\right)=d\left(\gamma_{j}^{-1} \gamma_{i} \cdot P, P\right) \geq \mu$, so that

$$
\gamma_{i} \cdot b \cap \gamma_{j} \cdot b=\emptyset \text { for all distinct indices } i \text { and } j \text { in }\{1, \ldots, r\} \text {. }
$$

From (5.3.10) and (5.3.11) it follows that $r \operatorname{vol} b \leq \operatorname{vol} B$. Combining this with (5.3.6), (5.3.7) and (5.3.8), we obtain

$$
\begin{aligned}
\frac{2\left(3^{N}-1\right)}{4 N+1} & <r \leq \frac{\operatorname{vol} B}{\operatorname{vol} b} \\
& \leq \pi(\sinh (2 N \lambda+\mu)-(2 N \lambda+\mu)) / 0.000589 \\
& <5334(\sinh (2 N \lambda+\mu)-(2 N \lambda+\mu)),
\end{aligned}
$$

which is a contradiction, since by definition (5.1.1) holds with $N=N(\lambda)$.

\section{From a Short Relation to a Volume Bound}

The main result of this section is Proposition 6.2. As we mentioned in the introduction, the basic method of proof of that proposition is due to Cooper [8]. Among the several preliminary results needed to apply Cooper's method in the present situation, Lemma 6.2 is the deepest, while Proposition 6.1 seems to be of particular independent interest.

Lemma 6.1. Let $N$ and $Q$ be irreducible, orientable 3-manifolds. Suppose that $\pi_{1}(N)$ is isomorphic to a subgroup of $\pi_{1}(Q)$ and that $N$ is closed. Then either $Q$ is closed or $N$ is simply connected.

Proof. First consider the case in which $\pi_{1}(N)$ is infinite. In this case $\pi_{1}(Q)$ is also infinite, and $Q$ and $N$ are aspherical by Proposition 3.6. Fix a base point $q$ in $Q$ and a subgroup $J$ of $\pi_{1}(Q, q)$ isomorphic to $\pi_{1}(N)$, and let $(\widetilde{Q}, \widetilde{q})$ denote the based covering of $(Q, q)$ determined by $J$. Since $N$ and $\widetilde{Q}$ are aspherical and have isomorphic fundamental groups, they are homotopy-equivalent. If $Q$ is not closed, then $\widetilde{Q}$ is not closed, and hence $H_{3}(\widetilde{Q} ; \mathbb{Z})=0$. But $H_{3}(N ; \mathbb{Z}) \neq 0$ since $N$ is closed, 
and we have a contradiction to the homotopy-equivalence of $\widetilde{Q}$ and $N$. Hence in this case $Q$ must be closed.

Now consider the case in which $\pi_{1}(N)$ is finite. In this case we will assume that $\pi_{1}(N)$ is non-trivial and show that $Q$ is closed, thus establishing the conclusion. The assumption that $\pi_{1}(N)$ is finite and non-trivial implies that $\pi_{1}(Q)$ has torsion, and so $Q$ cannot be aspherical [11, Lemma 8.4]. By Proposition [3.6, $\pi_{1}(Q)$ is finite. We apply Proposition 3.8, letting $Q$ play the role of $M$ in that proposition. This gives a compact, irreducible submanifold $Q_{0}$ of $Q$ such that the inclusion homomorphism $\pi_{1}\left(Q_{0}\right) \rightarrow \pi_{1}(Q)$ is an isomorphism. In particular, $\pi_{1}\left(Q_{0}\right)$ is finite, so that $H_{1}\left(Q_{0} ; \mathbb{Q}\right)=0$, and hence every component of $\partial Q_{0}$ is a sphere (for example by [32, Proposition 2.2]). Since $Q_{0}$ is irreducible it follows that either $\partial Q_{0}=\emptyset$ or $Q_{0}$ is a ball. In the latter case the hypothesis gives $\pi_{1}(N)=\{1\}$, a contradiction. Hence $\partial Q_{0}=\emptyset$, which implies that $Q=Q_{0}$ and that $Q$ is closed.

Proposition 6.1. Let $N$ and $Q$ be irreducible, orientable 3-manifolds (possibly with boundary). Suppose that $N$ is compact, that $\pi_{1}(N)$ is non-cyclic and that every component of $\partial N$ is a torus. Let $h: N \rightarrow Q$ be a map such that $h_{\sharp}: \pi_{1}(N) \rightarrow$ $\pi_{1}(Q)$ is injective. Then there exist a compact submanifold $Q_{0}$ of $Q$ such that every component of $\partial Q_{0}$ is a torus, and a map $h_{0}: N \rightarrow Q$ homotopic to $h$ such that $h_{0}(N) \subset Q_{0}$.

Proof. First note that since $N$ is irreducible, $\pi_{1}(N)$ is non-cyclic, and every component of $\partial N$ is a torus, it follows from Proposition 3.7 that $N$ is boundary-irreducible.

First consider the case in which $N$ is closed. Note that since $h_{\sharp}$ is injective, $\pi_{1}(N)$ is isomorphic to a subgroup of $\pi_{1}(Q)$. Since $\pi_{1}(N)$ is non-cyclic, it then follows from Lemma 6.1 that $Q$ is closed. Hence the conclusion holds in this case if we set $Q_{0}=Q$.

Next consider the case in which $\partial N \neq \emptyset$ and $Q$ is compact. Let us fix a Seifertfibered space $\Sigma \subset$ int $M$ having the property stated in Proposition 3.9. Let us denote by $J$ the union of all components $C$ of $\overline{Q-\Sigma}$ such that $\partial C \subset \partial \Sigma$.

In this case we will set $Q_{0}=J \cup \Sigma$, so that every component of $\partial Q_{0}$ is a torus, and we will construct a map $h_{0}: N \rightarrow Q$, homotopic to $h$, such that $h_{0}(N) \subset Q_{0}$.

Consider an arbitrary component $T$ of $\partial N$. By hypothesis $T$ is a torus. Since $N$ is boundary-irreducible, $T$ is $\pi_{1}$-injective in $N$. Since $h_{\sharp}: \pi_{1}(N) \rightarrow \pi_{1}(Q)$ is injective, it follows that $(h \mid T)_{\sharp}: \pi_{1}(T) \rightarrow \pi_{1}(Q)$ is injective. It then follows from the property of $\Sigma$ stated in Proposition 3.9 that $h \mid T: T \rightarrow Q$ is homotopic to a map whose image is contained in $\Sigma$. Since this is true for each component $T$ of $\partial N$, it follows that $h \mid \partial N$ is homotopic to a map $g: \partial N \rightarrow Q$ such that $g(\partial N) \subset \operatorname{int} \Sigma$. By the homotopy extension property of polyhedra, $g$ extends to a map from $N$ to $Q$ which is homotopic to $h$.

It follows from [15, Lemma 6.5] that we can choose an extension $h_{1}: N \rightarrow Q$ of $g$, homotopic to $h$, so that $h_{1}$ is transverse to $\partial \Sigma$ and so that each component of $h_{1}^{-1}(\partial \Sigma)$ is incompressible. Since $h_{1}(\partial N)=g(\partial N) \subset$ int $\Sigma$, we have $h_{1}^{-1}(\partial \Sigma) \subset$ $\operatorname{int} N$.

We claim:

6.1.1. If $K$ is a component of $h_{1}^{-1}\left(\overline{Q-Q_{0}}\right)$, then $h_{1} \mid K$ is homotopic rel $\partial K$ to a map $h_{K}$ with $h_{K}(K) \subset \partial \Sigma$. (In particular, $h_{1}(\partial K) \subset \partial \Sigma$.)

To prove Claim 6.1.1. consider a component $K$ of $h_{1}^{-1}\left(\overline{Q-Q_{0}}\right)$ and let $C$ denote the component of $\overline{Q-Q_{0}}$ containing $h_{1}(K)$. Since $h_{1}$ is transverse to $\partial \Sigma$ and maps 
$\partial N$ into int $\Sigma$, we have $h_{1}(\partial K) \subset C \cap \partial \Sigma$. In particular, $h_{1} \mid K: K \rightarrow C$ is a boundary-preserving map.

Every component of the frontier of $K$ in $N$ is a component of $h_{1}^{-1}(\partial \Sigma)$ and is therefore incompressible in $N$. Hence $K$ is $\pi_{1}$-injective in $N$. On the other hand, $h_{\sharp}: \pi_{1}(N) \rightarrow \pi_{1}(Q)$ is injective by hypothesis, and since $h_{1}$ and $h$ are homotopic, $\left(h_{1}\right)_{\sharp}: \pi_{1}(N) \rightarrow \pi_{1}(Q)$ is also injective. This shows that $\left(h_{1} \mid K\right)_{\sharp}: \pi_{1}(K) \rightarrow \pi_{1}(Q)$ is injective, and so in particular $\left(h_{1} \mid K\right)_{\sharp}: \pi_{1}(K) \rightarrow \pi_{1}(C)$ is injective.

Since $N$ is irreducible and boundary-irreducible, and since we have observed that the components of the frontier of $K$ in $N$ are incompressible, the manifold $K$ is also irreducible and boundary-irreducible. Since the components of $\partial N$ are tori and the components of the frontier of $K$ are incompressible, $\partial K$ has no sphere components. Since $\partial N \neq \emptyset$, we have $\partial K \neq \emptyset$. According to [15. Lemma 6.7], it follows that $K$ is a Haken manifold. Since $K$ is boundary-irreducible it is not a solid torus. It now follows from [15, Theorem 13.6] that every boundary-preserving map from $K$ to $C$ which induces an injection of fundamental groups is homotopic rel $\partial K$ either to a covering map or to a map whose image is contained in $\partial C$.

Suppose that $h_{1} \mid K: K \rightarrow C$ is homotopic rel $\partial K$ to a covering map. Then in particular we have $h(\partial K)=\partial C$. Since we have seen that $h_{1}(\partial K) \subset C \cap \partial \Sigma$, this implies that $\partial C \subset \partial \Sigma$, which by the definition of $Q_{0}$ implies that $C \subset Q_{0}$. This is a contradiction, since $C$ is a component of $\overline{Q-Q_{0}}$. Hence $h_{1} \mid K: K \rightarrow C$ is homotopic rel $\partial K$ to a map whose image is contained in $\partial C$. Since $h_{1}(\partial K) \subset$ $C \cap \partial \Sigma$, it follows that we in fact have $h_{K}(\partial K) \subset C \cap \partial \Sigma$. This proves Claim 6.1.1.

We may now define a map $h_{0}: N \rightarrow Q$ by letting $h_{0}$ agree with $h_{1}$ on $h_{1}^{-1}\left(Q_{0}\right)$ and setting $h_{0} \mid K=h_{K}$ for each component $K$ of $h_{1}^{-1}\left(\overline{Q-Q_{0}}\right)$. It is immediate from the properties of the $h_{K}$ stated in Claim 6.1.1 that $h_{0}$ is well defined, is homotopic to $h_{1}$ (and hence to $h$ ), and maps $N$ into $Q_{0}$. This establishes the conclusion in this case.

There remains the case in which $\partial N \neq \emptyset$ and $Q$ is non-compact. In this case, fix a compact submanifold $R_{0}$ of $Q$ containing $h(N)$. By Lemma 3.1, there is a compact irreducible submanifold $R$ of $M$ such that $R \supset R_{0}$. The hypotheses of the propositon continue to hold if $Q$ is replaced by $R$. As $R$ is compact, the case of the proposition already proved gives a compact submanifold $Q_{0}$ of $R \subset Q$ such that every component of $\partial Q_{0}$ is a torus, and a map $h_{0}: N \rightarrow R$ homotopic to $h$ in $R$ (and hence in $Q$ ) such that $h_{0}(N) \subset Q_{0}$.

Although it is convenient to state the following result in the hyperbolic setting, the proof is essentially topological.

Lemma 6.2. Let $M$ be a hyperbolic 3-manifold, let $K$ be a compact, path-connected space such that $\pi_{1}(K)$ has rank 2 and is not free, and let $f: K \rightarrow M$ be a continuous map such that $f_{\sharp}: \pi_{1}(K) \rightarrow \pi_{1}(M)$ is surjective and $f(K) \subset M$ is a polyhedron with respect to some $C^{1}$ triangulation of $M$. Then for each component $C$ of $M-f(K)$, the image of the inclusion homomorphism $\pi_{1}(C) \rightarrow \pi_{1}(M)$ is abelian.

Proof. We may assume that $\pi_{1}(M)$ is non-abelian, as otherwise the conclusion is obvious.

We fix a PL structure on $M$, defined by a $C^{1}$ triangulation, in which $f(K)$ is a polyhedron.

For the purpose of this proof, a compact polyhedron in $M$ will be termed "small" if it is contained in a 3-ball in $M$. Let $R$ be a regular neighborhood of $f(K)$ in 
$M$. Let $Q$ denote the union of $R$ with all small components of $\overline{M-R}$. Then no component of $\overline{M-Q}$ is small. If $S$ is any 2-sphere in $\operatorname{int} Q$, then $S$ bounds a 3-ball $B \subset M$, since $M$ is irreducible by assertion (1) of Proposition 3.5. If $B \not \subset Q$, then $B$ contains a component of $\overline{M-Q}$, which by definition must be small, a contradiction; hence $B \subset Q$. This shows that $Q$ is irreducible.

Fix a base point $x \in K$, and set $q=f(x) \in f(K) \subset R \subset Q$. We may regard $f$ as a map from the based space $(K, x)$ to the based space $(Q, q)$. Let $I \leq \pi_{1}(Q, q)$ denote the image of the homomorphism $f_{\sharp}: \pi_{1}(K, x) \rightarrow \pi_{1}(Q, q)$. Then $I$ determines a based covering space $p:(\widetilde{Q}, \widetilde{q}) \rightarrow(Q, q)$, and there is a unique lift $\widetilde{f}: K \rightarrow \widetilde{Q}$ such that $\widetilde{f}(x)=\widetilde{q}$. If $i: Q \rightarrow M$ denotes inclusion, we have a commutative diagram

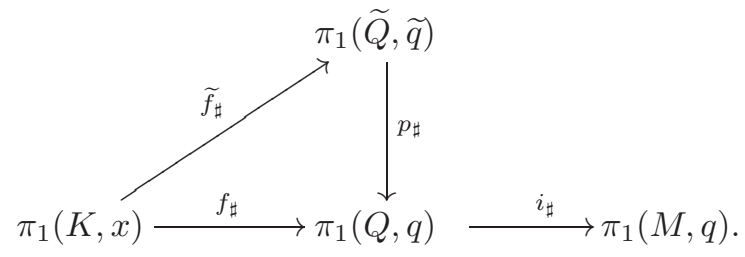

According to the hypothesis, $f_{\sharp} \circ i_{\sharp}$ is surjective, and hence by commutativity of the diagram, $i_{\sharp} \circ p_{\sharp}: \pi_{1}(\widetilde{Q}, \widetilde{q}) \rightarrow \pi_{1}(M, q)$ is surjective. Since $\pi_{1}(M)$ is non-abelian, it follows that $\pi_{1}(\widetilde{Q})$ is non-abelian, and in particular non-cyclic. On the other hand, the construction of $(\widetilde{Q}, \widetilde{q})$ implies that $\widetilde{f}_{\sharp}$ is surjective. Thus the non-cyclic group $\pi_{1}(\widetilde{Q})$ is a homomorphic image of $\pi_{1}(K)$, which by hypothesis is a non-free group of rank 2. It follows that $\pi_{1}(\widetilde{Q})$ is also a non-free group of rank exactly 2 .

Since $Q$ is irreducible, it follows from [23, p. 647, Theorem 3] that $\widetilde{Q}$ is irreducible. We now apply Proposition 3.8. letting $\widetilde{Q}$ play the role of $M$ in that proposition. This gives a compact, irreducible submanifold $N$ of $\widetilde{Q}$ such that the inclusion homomorphism $\pi_{1}(N) \rightarrow \pi_{1}(\widetilde{Q})$ is an isomorphism. In particular, $\pi_{1}(N)$ is a non-free group of rank exactly 2. Hence by Proposition 3.10, each component of $\partial N$ is a torus.

Set $h=p \mid N: N \rightarrow Q$. Since $p_{\sharp}: \pi_{1}(\widetilde{Q}) \rightarrow \pi_{1}(Q)$ is injective, and since the inclusion homomorphism $\pi_{1}(N) \rightarrow \pi_{1}(\widetilde{Q})$ is an isomorphism, the homomorphism $h_{\sharp} \rightarrow \pi_{1}(N) \rightarrow \pi_{1}(Q)$ is injective. We have seen that $N$ and $Q$ are compact, irreducible, orientable 3-manifolds, that $\pi_{1}(N)$ has rank 2 and is in particular noncyclic, and that every component of $\partial N$ is a torus. Thus all the hypotheses of Proposition 6.1 hold. The latter proposition now gives a compact submanifold $Q_{0}$ of $Q$ such that every component of $\partial Q_{0}$ is a torus, and a map $h_{0}: N \rightarrow Q$ homotopic to $h$, such that $h_{0}(N) \subset Q_{0}$.

We have seen that $i_{\sharp} \circ p_{\sharp}: \pi_{1}(\widetilde{Q}) \rightarrow \pi_{1}(M)$ is surjective. Since the inclusion homomorphism $\pi_{1}(N) \rightarrow \pi_{1}(\widetilde{Q})$ is an isomorphism, it follows that $i_{\sharp} \circ h_{\sharp}=$ $i_{\sharp} \circ(p \mid N)_{\sharp}: \pi_{1}(N) \rightarrow \pi_{1}(M)$ is surjective. Since the maps $h_{0}, h: N \rightarrow Q$ are homotopic, $i_{\sharp} \circ\left(h_{0}\right)_{\sharp}: \pi_{1}(N) \rightarrow \pi_{1}(M)$ is also surjective. Since $h_{0}(N) \subset Q_{0}$, the inclusion homomorphism $\pi_{1}\left(Q_{0}\right) \rightarrow M$ is surjective.

To establish the conclusion of the lemma, it suffices to show that for every component $c$ of $\overline{M-R}$, the image of the inclusion homomorphism $\pi_{1}(c) \rightarrow \pi_{1}(M)$ is abelian. According to the definition of $Q$, any such $c$ is either a small component of $\overline{M-R}$, in which case the image of the inclusion homomorphism is trivial, or 
a component of $\overline{M-Q}$. In the latter case, $c$ is contained in a component $c_{0}$ of $\overline{M-Q_{0}}$, and we shall complete the proof by showing that the image of the inclusion homomorphism $\pi_{1}\left(c_{0}\right) \rightarrow \pi_{1}(M)$ is abelian. Let us fix any component $T$ of $\partial c_{0}$. Then $T$ is a component of $\partial Q_{0}$ and is therefore a torus. It follows from assertions (2) and (3) of Proposition 3.5 that $T$ is the boundary of a 3-dimensional submanifold $J$ of $M$, closed as a subset of $M$, such that the inclusion homomorphism $\pi_{1}(J) \rightarrow$ $\pi_{1}(M)$ has abelian image. Since $Q_{0}$ is connected, we must have either $J=c_{0}$, in which case the required conclusion holds, or $J \supset Q_{0}$. The latter alternative would imply that the inclusion homomorphism $\pi_{1}\left(Q_{0}\right) \rightarrow \pi_{1}(M)$ has abelian image. This is impossible, as this inclusion homomorphism is surjective and $\pi_{1}(M)$ is nonabelian.

Lemma 6.3. Let $M$ be a hyperbolic 3-manifold of finite volume, and let $C$ be a compact 3-dimensional (smooth) submanifold of $M$. Suppose that the inclusion homomorphism $\pi_{1}(C) \rightarrow \pi_{1}(M)$ has abelian image. Then

$$
\operatorname{vol} C \leq \frac{1}{2} \text { area } \partial C
$$

Proof. This is essentially Lemma 4.4 of [3]. The authors of [3] assume $C$ to be "PL" in a sense that is not clear, but their proof can be read in the smooth category without change.

Lemma 6.4. Let $M$ be a hyperbolic 3-manifold of finite volume. Let $X \subset M$ be a compact set which is a 2-dimensional polyhedron with respect to some smooth triangulation of $M$. Suppose that for every component $C$ of $M-X$, the inclusion homomorphism $\pi_{1}(C) \rightarrow \pi_{1}(M)$ has abelian image. Then

$$
\operatorname{vol} M \leq \operatorname{area} X .
$$

Proof. Let $m$ denote the number of cusps of $M$. Let $\epsilon>0$ be given. Since $M$ has finite volume, there are disjoint $\mathbb{Z} \times \mathbb{Z}$-cusp neighborhoods $V_{1}, \ldots, V_{m}$ in $M$, for some $m>0$, such that $M_{0}:=\overline{M-\left(V_{1} \cup \cdots \cup V_{m}\right)}$ is compact. Set $V=V_{1} \cup \cdots \cup V_{m}$. After replacing the $V_{i}$ by smaller $\mathbb{Z} \times \mathbb{Z}$-cusp neighborhoods if necessary, we may assume that each $V_{i}$ has volume less than $\epsilon / m$, that each $\partial V_{i}$ has area less than $\epsilon / m$, and that the $V_{i}$ are disjoint from $X$. Hence vol $V<\epsilon$, area $\partial V<\epsilon$, and $X \subset \operatorname{int} M_{0}$.

Since $X$ is a 2-dimensional polyhedron with respect to the given smooth triangulation of $M$, there is an open neighborhood $U$ of $X$ in int $M_{0}$ with vol $U<\epsilon$. Let $N \subset U$ be a smooth regular neighborhood of $X$ in the sense of [16]. We may choose $N$ in such a way that area $\partial N<\epsilon+2$ area $X$.

Let $C_{1}, \ldots, C_{n}$ denote the components of $\overline{M_{0}-N}$. Then each $C_{j}$ is contained in a component of $M-X$, and hence the inclusion homomorphism $\pi_{1}\left(C_{j}\right) \rightarrow \pi_{1}(M)$ has abelian image. According to Lemma 6.3. we have

$$
\operatorname{vol} C_{j} \leq \frac{1}{2} \text { area } \partial C_{j}
$$

for $j=1, \ldots, n$. Hence

$$
\begin{aligned}
\operatorname{vol}\left(\overline{M_{0}-N}\right) & \leq \frac{1}{2} \text { area } \partial\left(\overline{M_{0}-N}\right) \\
& =\frac{1}{2} \text { area } \partial M_{0}+\frac{1}{2} \text { area } \partial N .
\end{aligned}
$$


Since area $\partial M_{0}=$ area $\partial V<\epsilon$ and area $\partial N<\epsilon+2$ area $X$, it follows that

$$
\operatorname{vol}\left(\overline{M_{0}-N}\right)<\epsilon+\operatorname{area} X \text {. }
$$

Now since $M=V \cup N \cup \overline{M_{0}-N}$, we have

$$
\begin{aligned}
\operatorname{vol} M & =\operatorname{vol} V+\operatorname{vol} N+\operatorname{vol}\left(\overline{M_{0}-N}\right) \\
& <\epsilon+\epsilon+(\epsilon+\operatorname{area} X) \\
& =3 \epsilon+\text { area } X .
\end{aligned}
$$

Since $\epsilon>0$ was arbitrary, it follows that vol $M \leq$ area $X$.

Proposition 6.2. Let $(M, \star)$ be a based closed, orientable, hyperbolic 3-manifold such that $\pi_{1}(M, \star)$ is generated by two non-commuting elements $x$ and $y$. Let $\lambda>0$ be given, and suppose that $x$ and $y$ are represented by closed loops of length $<\lambda$ based at $\star$. Let $W$ be a non-trivial reduced word in two letters such that $W(x, y)=1$. Then

(In particular, $M$ has finite volume.)

$$
\operatorname{vol} M<(\text { length }(W)-2) \min (\pi, \lambda) .
$$

dja

Proof. If $M$ has infinite volume, then Proposition 3.12 implies that the non-commuting elements $x$ and $y$ cannot both be represented by loops of length $<\log 3$ based at $\star$. Hence the hypothesis implies that vol $M<\infty$.

Since $x$ and $y$ do not commute, they are in particular both non-trivial elements of $\pi_{1}(M, \star)$. Since $\pi_{1}(M)$ is generated by $x$ and $y$, it is non-abelian and in particular non-cyclic. As the fundamental group of a hyperbolic manifold, it is also torsionfree, and hence $x$ and $y$ have infinite order.

We will regard $W$ as a word in two abstract generators $\xi$ and $\eta$. Let us write $W=\psi_{1} \cdots \psi_{n}$, where each $\psi_{j}$ has the form $\xi^{ \pm 1}$ or $\eta^{ \pm 1}$. We may also identify $W$ with a non-trivial element of the free group $F$ on the generators $x$ and $y$. Since $\pi_{1}(M)$ is generated by $x$ and $y$ and since $W(x, y)=1$, there is an epimorphism from $F /\langle\langle W\rangle\rangle$ to $\pi_{1}(M, \star)$ that maps $\xi$ to $x$ and $\eta$ to $y$. Set $n=$ length $W$.

If there is a non-trivial reduced word $W^{\prime}$ in two letters such that $n^{\prime}:=$ length $W^{\prime}$ $<n$ and such that $W^{\prime}(x, y)=1$, and if vol $M<\left(n^{\prime}-2\right) \min (\pi, \lambda)$, then in particular $\operatorname{vol} M<(n-2) \min (\pi, \lambda)$. Hence, arguing inductively, we may assume that $n$ is the minimal length of any non-trivial reduced word which gives a relation between $x$ and $y$.

If $n$ were at most 3 , either $F /\langle\langle W\rangle\rangle$ would be cyclic, which is impossible since $\pi_{1}(M)$ is non-cyclic, or the image of $\xi$ or $\eta$ in $F /\langle\langle W\rangle\rangle$ would have finite order, which is impossible since $x$ and $y$ have infinite order. Hence $n \geq 4$.

The hypothesis implies that there are loops $\alpha, \beta:[0,1] \rightarrow M$ based at $\star$ such that $\alpha \mid(0,1)$ and $\beta \mid(0,1)$ are open geodesics of length $<\lambda$ and such that $[\alpha]=x$ and $[\beta]=y$. These geodesics are non-constant since $x$ and $y$ are non-trivial elements of $\pi_{1}(M, \star)$. Let $\mathcal{G}$ be a graph with one vertex $v$ and two closed edges $a$ and $b$. Fix loops $\kappa_{a}$ and $\kappa_{b}$ in $\mathcal{G}$, based at $v$, such that $\kappa_{a} \mid(0,1)$ and $\kappa_{b} \mid(0,1)$ are homeomorphisms of $(0,1)$ onto int $a$ and int $b$, respectively. Define a continuous map $\phi: \mathcal{G} \rightarrow M$ by setting $\phi(v)=\star, \phi \mid$ int $a=\alpha \circ \kappa_{a}^{-1}$ and $\phi \mid \operatorname{int} b=\beta \circ \kappa_{b}^{-1}$.

Let us define a map $\omega: S^{1} \rightarrow \mathcal{G}$ as follows. Fix $n$ points on $S^{1}$, labeled in counterclockwise order as $\zeta_{0}, \ldots, \zeta_{n-1}$. Set $\zeta_{n}=\zeta_{0}$. For $1 \leq j \leq n$ let $A_{j}$ denote the arc which, when oriented counterclockwise, has $\zeta_{j-1}$ and $\zeta_{j}$ as its initial and 
terminal points, respectively. Fix a homeomorphism $h_{j}: A_{j} \rightarrow[0,1]$ which maps $\zeta_{j-1}$ and $\zeta_{j}$ to 0 and 1 , respectively. Set $\omega\left(\zeta_{j}\right)=\star$ for $0 \leq j \leq n-1$. For $j=1, \ldots, n$, define $\omega \mid A_{i}$ to be $\kappa_{a} \circ h_{j}, \overline{\kappa_{a}} \circ h_{j}, \kappa_{b} \circ h_{j}$, or $\overline{\kappa_{b}} \circ h_{j}$, according to whether $\psi_{j}$ is equal to $\xi, \xi^{-1}, \eta$ or $\eta^{-1}$, respectively.

Since $W(x, y)=1$, the map $\phi \circ \omega$ is homotopic to a constant. Hence if we define a CW complex $K$ by attaching a 2-cell to $\mathcal{G}$ via the attaching map $\omega$, then $\phi$ extends to a map $f: K \rightarrow M$. Let $c: D^{2} \rightarrow K$ denote the characteristic map for the 2-cell of $K$. For each $j$ with $2 \leq j \leq n-2$, let $\sigma_{j}$ denote the line segment in the Euclidean disk $D^{2}$ with endpoints $\zeta_{0}$ and $\zeta_{j}$. Set $\sigma_{1}=A_{1}$ and $\sigma_{n-1}=A_{n}$. Then for each $j$ with $1 \leq j \leq n$, the topological arc $\sigma_{j}$ has endpoints $\zeta_{0}$ and $\zeta_{j}$. For $j=1, \ldots, n-1$, by precomposing $f \circ c \mid \sigma_{j}$ with a homeomorphism from $[0,1]$ to $\sigma_{i}$ that maps 0 to $\zeta_{0}$ and 1 to $\zeta_{j}$, we obtain a loop $\nu_{j}$ based at $\star$. We have $\left[\nu_{j}\right]=W^{\prime}(x, y)$, where $W^{\prime}:=\psi_{1} \cdots \psi_{j}$ is a reduced word of length $j<n$. By our mimimality assumption on $n$, it follows that $\nu_{j}$ is a homotopically nontrivial loop and is therefore fixed-endpoint homotopic to a loop whose restriction to $(0,1)$ is a non-constant geodesic. Furthermore, each of the paths $\nu_{1} \mid(0,1)$ and $\nu_{n} \mid(0,1)$ is a (possibly orientation-reversing) reparametrization of either $\alpha \mid(0,1)$ or $\beta \mid(0,1)$, and is therefore a non-constant geodesic. Hence after modifying $f$ within its homotopy class rel $\mathcal{G}$, we may assume that $\nu_{j} \mid(0,1)$ is a non-constant geodesic for $j=1, \ldots, n-1$.

The set $D^{2}-\left(\sigma_{1} \cup \cdots \cup \sigma_{n-1}\right)$ has $n-2$ components. The closures of these components are topological disks, which we may label as $\tau_{1}, \ldots, \tau_{n-2}$, where $\partial \tau_{j}=$ $\sigma_{j} \cup A_{j+1} \cup \sigma_{j+1}$.

Let $j$ be any index with $1 \leq j \leq n-2$. The maps $f \mid \sigma_{j}$ and $f \mid \sigma_{j+1}$ are reparametrizations of the non-constant geodesic paths $\nu_{j}$ and $\nu_{j+1}$, while $f \mid A_{j+1}$ may be obtained from one of the non-constant geodesic paths $\alpha$ or $\beta$ by precomposing it with some homeomorphism from $A_{j+1}$ to $[0,1]$. Hence if $\widetilde{G}_{j}$ is a lift of the map $f \circ c \mid \tau_{j}$ to $\mathbb{H}^{3}$, then $\widetilde{G}_{j}\left(\partial \tau_{j}\right)$ is the boundary of a triangle $T_{j} \subset \mathbb{H}^{3}$. Since one of the sides of $T_{j}$ is a lift of either $\alpha$ or $\beta$, the length of the shortest side of $T_{j}$ is less than $\lambda$. Hence it follows from Proposition 2.2 that area $T_{j}<\min (\pi, \lambda)$.

Since $M$ is aspherical, we may arrange after modifying $f$ by a homotopy rel $\mathcal{G} \cup c\left(\sigma_{2}\right) \cup \cdots \cup c\left(\sigma_{n-2}\right)$ that for each $j$ with $1 \leq j \leq n-1$, the map $f \mid \tau_{j}$ admits a lift to $\mathbb{H}^{3}$ which maps $\tau_{j}$ onto $T_{j}$. Now by Corollary 3.3 we may fix a smooth triangulation of $M$ with respect to which each of the sets $\alpha([0,1]), \beta([0,1])$ and $f \circ c\left(\tau_{j}\right)=p \circ \widetilde{G}_{j}\left(\tau_{j}\right)(j=1, \ldots, n-2)$, is polyhedral. It follows that the union $f(K)$ of these sets is also polyhedral. We have

$$
\operatorname{area}\left(f \circ c\left(\tau_{j}\right)\right) \leq \operatorname{area} T_{j}<\min (\pi, \lambda) .
$$

Hence

$$
\text { area } f(K) \leq \sum_{j=1}^{n-2} \operatorname{area}\left(f \circ c\left(\tau_{j}\right)\right)<(n-2) \min (\pi, \lambda) .
$$

According to the construction of $K$ we have $\pi_{1}(K) \cong F /\langle\langle W\rangle\rangle$, where, as above, $F$ denotes the free group on the generators $\xi$ and $\eta$. In particular, $\pi_{1}(K)$ has rank at most 2. Furthermore, since $W$ is a non-trivial reduced word, $\pi_{1}(K)$ is not a free group of rank 2 . On the other hand, since $x$ and $y$ generate $\pi_{1}(M, \star)$, the map $f_{\sharp}: \pi_{1}(K, v) \rightarrow \pi_{1}(M, \star)$ is surjective. Since we have observed that $\pi_{1}(M)$ is non-cyclic, $\pi_{1}(K)$ is also non-cyclic; hence $\pi_{1}(K)$ has rank exactly 2 . Thus all the 
hypotheses of Lemma 6.2 are seen to hold, and it follows that for each component $C$ of $M-f(K)$, the image of the inclusion homomorphism $\pi_{1}(C) \rightarrow \pi_{1}(M)$ is abelian.

We may now apply Lemma 6.4, taking $X=f(K)$, to deduce that

$$
\operatorname{vol} M \leq \text { area } f(K) \text {. }
$$

The required conclusion vol $M<(n-2) \min (\pi, \lambda)$ follows immediately from 6.2.1) and (6.2.2).

\section{A CONCRETE BOUND FOR vol $M$ WHEN $\mu(M)<(\log 3) / 2$, AND ITS CONSEQUENCES}

Theorem 7.1. Let $\lambda$ be a positive real number strictly less than $(\log 3) / 2$, and let $N(\lambda)$ be defined by Notation 5.1. Then for every orientable hyperbolic 3-manifold $M$ with $\mu(M)<\lambda$ we have

$$
\operatorname{vol} M<\lambda \cdot(8 N(\lambda)-2) .
$$

Proof. Suppose that $M$ is an orientable hyperbolic 3-manifold such that $\mu(M)<$ $\lambda$, i.e., such that $\lambda$ is not a Margulis number for $M$. Let us write $M=\mathbb{H}^{3} / \Gamma$, where $\Gamma \leq \operatorname{Isom}_{+}\left(\mathbb{H}^{3}\right)$ is discrete and torsion-free. Then there are non-commuting elements $x$ and $y$ of $\Gamma$ such that $\max (d(P, x \cdot P), d(P, y \cdot P))<\lambda$ for $i=1,2$. According to Proposition 5.3 there is a reduced word $W$ in two letters, with $0<$ length $W \leq 8 N(\lambda)$, such that $W(x, y)=1$.

Set $\widetilde{\Gamma}=\langle x, y\rangle \leq \Gamma$ and $\widetilde{M}=\mathbb{H}^{3} / \widetilde{\Gamma}$. Let $\star \in \widetilde{M}$ denote the image of $P \in \mathbb{H}^{3}$ under the quotient map $\mathbb{H}^{3} \rightarrow \mathbb{H}^{3} / \widetilde{\Gamma}$. Then $\left(\mathbb{H}^{3}, P\right)$ is a based covering space of $(\widetilde{M}, \star)$. Under the natural identification of $\pi_{1}(\widetilde{M}, \star)$ with the deck group $\widetilde{\Gamma}$, the elements $x$ and $y$ are identified with generators of $\pi_{1}(\widetilde{M}, \star)$ which are represented by closed loops of length $<\lambda$ based at $\star$. Applying Proposition 6.2. with $\widetilde{M}$ playing the role of $M$ in that proposition, we deduce that

$$
\operatorname{vol} \widetilde{M}<\lambda(\operatorname{length}(W)-2) \leq \lambda(8 N(\lambda)-2) .
$$

(In particular $\widetilde{M}$ has finite volume.) Since $\widetilde{M}$ covers $M$, it follows that

$$
\operatorname{vol} M<\lambda(8 N(\lambda)-2) \text {. }
$$

In the following corollary, $V_{0}=0.94 \ldots$ will denote the volume of the Weeks manifold [36].

Corollary 7.1. Let $\lambda$ be a positive real number strictly less than $(\log 3) / 2$, and let $N(\lambda)$ be defined by Notation 5.1. Then for every orientable hyperbolic 3-manifold $M$, with $\mu(M)<\lambda$, the group $\pi_{1}(M)$ has a rank-2 subgroup of index at most $\lambda \cdot(8 N(\lambda)-2) / V_{0}$.

Proof. Suppose that $M$ is a closed, orientable hyperbolic 3-manifold such that $\mu(M)<\lambda$, i.e., such that $\lambda$ is not a Margulis number for $M$. Write $M_{\infty}=\mathbb{H}^{3} / \Gamma$, where $\Gamma \leq \operatorname{Isom}_{+}\left(\mathbb{H}^{3}\right)$ is discrete and torsion-free. Then by definition there exist a point $P \in \mathbb{H}^{3}$ and non-commuting elements $x, y \in \Gamma$ such that

$$
\max (d(P, x \cdot P), d(P, y \cdot P))<\lambda .
$$

Now $\widetilde{\Gamma}:=\langle x, y\rangle$ is a non-abelian rank-2 subgroup of $\Gamma$. 
Since $x$ and $y$ are non-commuting elements of $\widetilde{\Gamma}$, it follows from (7.1.1) that $\lambda$ is not a Margulis number for $\widetilde{M}$, i.e., that $\mu(\widetilde{M})<\lambda$. Hence by Theorem 7.1 we have vol $\widetilde{M}<\lambda \cdot(8 N(\lambda)-2)$. (In particular, $\widetilde{M}$ has finite volume.) On the other hand, it is shown in [25] that the Weeks manifold has minimal volume among all orientable hyperbolic 3-manifolds. Hence vol $M \geq V_{0}$. We therefore have

$$
[\Gamma: \widetilde{\Gamma}]=\frac{\operatorname{vol} \widetilde{M}}{\operatorname{vol} M}<\frac{\lambda \cdot(8 N(\lambda)-2)}{V_{0}} .
$$

Consequently, it follows that $\pi_{1}(M) \cong \Gamma$ has a rank-2 subgroup of index at most $\lambda \cdot(8 N(\lambda)-2) / V_{0}$.

Corollary 7.2. Let $\lambda$ be a positive real number strictly less than $(\log 3) / 2$. Then for every hyperbolic 3-manifold $M$ with $\mu(M)<\lambda$, we have

$$
\operatorname{rank} \pi_{1}(M) \leq 2+\log _{2}\left(\lambda \cdot(8 N(\lambda)-2) / V_{0}\right) .
$$

Proof. Let $M$ be an orientable hyperbolic 3-manifold with $\mu(M)<\lambda$. According to Corollary $7.1 \pi_{1}(M)$ has a rank-2 subgroup $X$ of index at most $\lambda \cdot(8 N(\lambda)-2) / V_{0}$. According to Proposition 2.1, we have

$$
\operatorname{rank} \pi_{1}(M) \leq \operatorname{rank} X+\log _{2}\left[\pi_{1}(M): X\right] \leq 2+\log _{2}\left(\frac{\lambda \cdot(8 N(\lambda)-2)}{V_{0}}\right) .
$$

We will conclude with three corollaries which follow immediately upon combining the earlier results of this section with the estimate for $N(\lambda)$ given by Proposition 5.2 and Meyerhoff's lower bound [24] of 0.104 for $\mu_{+}(3)$. The first, Corollary 7.3 , was stated in the introduction as Theorem B, and the other two were presented as corollaries to Theorem B (although we will derive them formally from Corollaries 7.1 and 7.2 above).

Corollary 7.3. Let $\lambda$ be a positive real number strictly less than $(\log 3) / 2$. Then for every orientable hyperbolic 3-manifold $M$ with $\mu(M)<\lambda$ we have

$$
\operatorname{vol} M<\lambda\left(6+\frac{880}{\log 3-2 \lambda} \log \frac{1}{\log 3-2 \lambda}\right) .
$$

Proof. Since $\lambda>\mu(M)$, we have in particular that $\lambda>\mu_{+}(3)>0.1$. The assertion now follows from Theorem 7.1 and Proposition 5.2 .

Corollary 7.4. Let $\lambda$ be a positive real number strictly less than $(\log 3) / 2$. Then for every orientable hyperbolic 3-manifold $M$ with $\mu(M)<\lambda$, the group $\pi_{1}(M)$ has a rank-2 subgroup of index at most

$$
\frac{\lambda}{V_{0}}\left(6+\frac{880}{\log 3-2 \lambda} \log \frac{1}{\log 3-2 \lambda}\right) .
$$

Proof. Since $\lambda>\mu(M)$, we have in particular that $\lambda>\mu_{+}(3)>0.1$. The assertion now follows from Corollary 7.1 and Proposition 5.2 .

Corollary 7.5. Let $\lambda$ be a positive real number strictly less than $(\log 3) / 2$. Then for every hyperbolic 3-manifold $M$ with $\mu(M)<\lambda$, we have

$$
\operatorname{rank} \pi_{1}(M) \leq 2+\log _{2}\left(\frac{\lambda}{V_{0}}\left(6+\frac{880}{\log 3-2 \lambda} \log \frac{1}{\log 3-2 \lambda}\right)\right) .
$$


Proof. Since $\lambda>\mu(M)$, we have in particular that $\lambda>\mu_{+}(3)>0.1$. The assertion now follows from Corollary 7.2 and Proposition 5.2 .

\section{REFERENCES}

1. Ian Agol. Tameness of hyperbolic 3-manifolds. arXiv:math.GT/0405568.

2. Ian Agol, Marc Culler, and Peter B. Shalen. Dehn surgery, homology and hyperbolic volume. Algebr. Geom. Topol., 6:2297-2312, 2006. MR2286027(2008f:57024)

3. Ian Agol and Yi Liu. Presentation length and Simon's conjecture. arXiv:1006.5262.

4. James W. Anderson, Richard D. Canary, Marc Culler, and Peter B. Shalen. Free Kleinian groups and volumes of hyperbolic 3-manifolds. J. Differential Geom., 43(4):738-782, 1996. MR:1412683 (98c:57012)

5. Riccardo Benedetti and Carlo Petronio. Lectures on hyperbolic geometry. Universitext. Springer-Verlag, Berlin, 1992. MR1219310(94e:57015)

6. Danny Calegari and David Gabai. Shrinkwrapping and the taming of hyperbolic 3-manifolds. J. Amer. Math. Soc., 19(2):385-446 (electronic), 2006. MR2188131 (2006g:57030)

7. R. D. Canary, D. B. A. Epstein, and P. L. Green. Notes on notes of Thurston [MR0903850]. In Fundamentals of hyperbolic geometry: Selected expositions, volume 328 of London Math. Soc. Lecture Note Ser., pages 1-115. Cambridge Univ. Press, Cambridge, 2006. With a new foreword by Canary. MR 2235710

8. Daryl Cooper. The volume of a closed hyperbolic 3-manifold is bounded by $\pi$ times the length of any presentation of its fundamental group. Proc. Amer. Math. Soc., 127(3):941-942, 1999. MR.1646313(99i:57025)

9. Marc Culler and Peter B. Shalen. Margulis numbers for Haken manifolds. Accepted Israel Journal of Mathematics.

10. Marc Culler and Peter B. Shalen. Paradoxical decompositions, 2-generator Kleinian groups, and volumes of hyperbolic 3-manifolds. J. Amer. Math. Soc., 5(2):231-288, 1992. MR.1135928 (93a:57017)

11. D. B. A. Epstein. Projective planes in 3-manifolds. Proc. London Math. Soc. (3), 11:469-484, 1961. MR0152997 (27:2968)

12. R. Mark Goresky. Triangulation of stratified objects. Proc. Amer. Math. Soc., 72(1):193-200, 1978. MR0500991 (58:18473)

13. Hans Grauert. On Levi's problem and the imbedding of real-analytic manifolds. Ann. of Math. (2), 68:460-472, 1958. MR0098847(20:5299)

14. Jack E. Graver. An analytic triangulation of an arbitrary real analytic variety. J. Math. Mech., 13:1021-1036, 1964. MR0167989 (29:5254)

15. John Hempel. 3-manifolds. AMS Chelsea Publishing, Providence, RI, 2004. Reprint of the 1976 original. MR2098385 (2005e:57053)

16. Morris W. Hirsch. Smooth regular neighborhoods. Ann. of Math. (2), 76:524-530, 1962. MR0149492(26:6979)

17. William H. Jaco and Peter B. Shalen. Seifert fibered spaces in 3-manifolds. Mem. Amer. Math. Soc., 21(220):viii+192, 1979. MR.539411(81c:57010)

18. Klaus Johannson. Homotopy equivalences of 3-manifolds with boundaries, volume 761 of Lecture Notes in Mathematics. Springer, Berlin, 1979. MR551744 (82c:57005)

19. T. Jørgensen and A. Marden. Algebraic and geometric convergence of Kleinian groups. Math. Scand., 66(1):47-72, 1990. MR:1060898 (91f:30068)

20. Troels Jørgensen. On discrete groups of Möbius transformations. Amer. J. Math., 98(3):739749, 1976. MR0427627(55:658)

21. Troels Jørgensen and Peter Klein. Algebraic convergence of finitely generated Kleinian groups. Quart. J. Math. Oxford Ser. (2), 33(131):325-332, 1982. MR668178 (83j:30050)

22. Wilhelm Magnus. Über freie Faktorgruppen und freie Untergruppen gegebener Gruppen. Monatsh. Math. Phys., 47(1):307-313, 1939. MR.1550819

23. William Meeks III, Leon Simon, and Shing Tung Yau. Embedded minimal surfaces, exotic spheres, and manifolds with positive Ricci curvature. Ann. of Math. (2), 116(3):621-659, 1982. MR678484(84f:53053)

24. Robert Meyerhoff. A lower bound for the volume of hyperbolic 3-manifolds. Canad. J. Math., 39(5):1038-1056, 1987. MR918586 (88k:57049) 
25. Peter Milley. Minimum volume hyperbolic 3-manifolds. J. Topol., 2(1):181-192, 2009. MR2499442(2010d:57018)

26. Edwin E. Moise. Affine structures in 3-manifolds. II. Positional properties of 2-spheres. Ann. of Math. (2), 55:172-176, 1952. MR0045380(13:574a)

27. James Munkres. Obstructions to the smoothing of piecewise-differentiable homeomorphisms. Ann. of Math. (2), 72:521-554, 1960. MR0121804 (22:12534)

28. H. Namazi and J. Souto. Nonrealizability in handlebodies and ending laminations. preprint.

29. Ken'ichi Ohshika. Realizing end invariants by limits of minimally parabolic, geometrically finite groups. arXiv:math.DG/0504506.

30. G. P. Scott. Compact submanifolds of 3-manifolds. J. London Math. Soc. (2), 7:246-250, 1973. MR0326737 (48:5080)

31. Caroline Series. A crash course on Kleinian groups. Rend. Istit. Mat. Univ. Trieste, 37(1-2):138 (2006), 2005. MR2227047(2006m:30075)

32. Peter B. Shalen. A generic Margulis number for hyperbolic 3-manifolds. arXiv:1008.4081.

33. Peter B. Shalen. Trace fields and Margulis numbers. In preparation.

34. Peter B. Shalen and Philip Wagreich. Growth rates, $Z_{p}$-homology, and volumes of hyperbolic 3-manifolds. Trans. Amer. Math. Soc., 331(2):895-917, 1992. MR1156298 (93d:57002)

35. Friedhelm Waldhausen. On irreducible 3-manifolds which are sufficiently large. Ann. of Math. (2), 87:56-88, 1968. MR0224099(36:7146)

36. Jeffrey Weeks. Hyperbolic structures on 3-manifolds. PhD thesis, Princeton Univ., 1985. MR2634492

37. J. H. C. Whitehead. Manifolds with transverse fields in euclidean space. Ann. of Math. (2), 73:154-212, 1961. MR0124917(23:A2225)

Department of Mathematics, Statistics, and Computer Science (M/C 249), University of Illinois at Chicago, 851 S. Morgan Street, Chicago, Illinois 60607-7045

E-mail address: shalen@math.uic.edu 